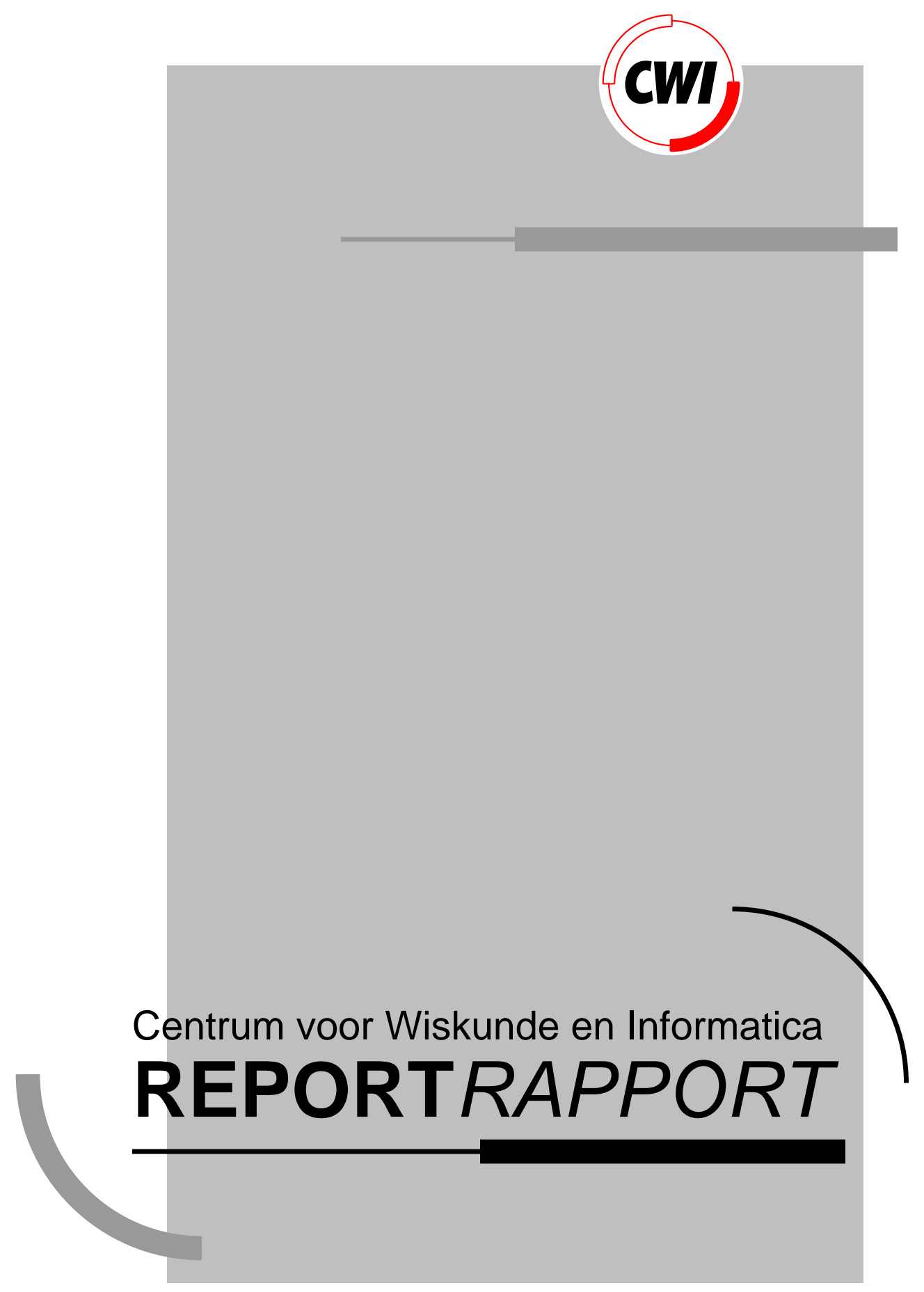

Optimal Multi-Writer Multi-Reader Atomic Register

A. Israeli, A. Shaham

Computer Science/Department of Algorithmics and Architecture

CS-R9235 1992 



\title{
Optimal Multi-Writer Multi-Reader Atomic Register
}

\author{
Amos Israeli \\ CWI \\ P.O. Box 4079, $1009 A B$ \\ Amsterdam, The Netherlands \\ $A N D$ \\ Dept. of Electrical Engineering \\ Technion - Israel \\ Amnon Shaham \\ CWI \\ P.O. Box 4079, 1009 AB \\ Amsterdam, The Netherlands \\ AND \\ Dept. of Computer Science \\ Technion - Israel
}

\begin{abstract}
This paper addresses the wide gap in space complexity of atomic, multi-writer, multireader register implementations. While the space complexity of all previous implementations is linear, the lower bounds are logarithmic. We present two implementations which close this gap: The first implementation uses multi-reader physical registers while the second uses single-reader physical registers. Both implementations are optimal with respect to the two most important complexity criteria: Their space complexity is logarithmic and their time complexity is linear.
\end{abstract}

1991 Mathematics Subject Classification: 68M10,68Q22, 68Q25.

CR Categories: B.3.2, B.4.3, D.4.1, D.4.4.

Keywords and Phrases: Shared Register, Concurrent Reading and Writind, Atomicity, Multiwriter Register.

Note: This work is partially supported by NWO through NFI Project ALADDIN under Contract number NF 62-376. A preliminary version of this paper was presented in the 11th Annual ACM Symposium on Principles of Distributed Computing, August 1992, Vancouver, Canada.

\section{INTRODUCTION}

At the most basic level of interprocessor communication, data is transferred via registers - memory devices which support read and write operations. Each register can store any element from its set of permitted values; it has a set of writers - processors that can write values in the register, and a set of readers - processors that can read values from the register. A write operation takes some permitted value as a parameter and stores it in the register; a read operation returns a (permitted) value stored in the register. The stored and returned values should satisfy some consistency guarantees 
which depend on the type of the register. Lamport in [La86a, La86b] was the first to formalize the notion of a register. He defined three types of registers in terms of the consistency guarantees they provide: safe, regular and atomic. An atomic register supports read and write as atomic, indivisible, operations. Safe and regular registers provide inferior consistency guarantees which are not discussed in this paper. The execution of an operation is called an action. Under the global time model, which we assume throughout this paper, each action has a starting time and an ending time. The interval between the starting and ending times of an action $a$ is called the execution interval of $a$. Each writer or reader executes actions in a serial manner, but we assume absolutely no synchronization among different processors, thus actions of distinct processors may be executed in overlapping time-periods.

In a situation in which some specific type of register is not available at the local hardware store, one may resort to implementing the required register using some available registers. For the user, such implementation is a black box which behaves exactly according to its specifications. Formally, an implementation of a logical register using a set of physical registers, consists of a hardware arrangement of the physical registers and two programs that are called the writer protocol and the reader protocol. Both programs are composed of operations of the physical registers (which are called physical operations) and constitute the operations of the logical register (which are called the logical operations). The set of processors is partitioned into logical writers and logical readers, that is, writers and readers of the logical register. For simplicity we assume that each processor is either a logical writer or a logical reader though in reality a processor can function as both.

An implementation is correct if the assumption that all physical registers provide their consistency guarantees implies that every execution of the logical register satisfies its consistency guarantees. For an atomic logical register this requirement materializes as follows: For every logical execution $E$, there exists an assignment of a serialization time for every logical action in $E$, that induces a serialization of all logical actions in $E$. Under this serialization every read action returns the value written by the write action which is the most recent preceding write. To avoid trivial solutions it is required that the reader and writer protocols are wait-free and that each action is serialized within its execution interval.

Throughout this paper $w$ and $r$ are used for the number of writers and readers, respectively, and $n=w+r$ is the total number of processors in the system. A register with $w$ writers and $r$ readers is denoted as a $(w, r)$-register. In [La86a, La86b] Lamport presented five implementations of various logical registers with a single writer. When some of these implementations are combined, they form an implementation of an atomic $(1,1)$-register with any value set, from binary, safe, $(1,1)$-registers. Several papers, motivated by the work of Lamport, studied the intriguing problem of implementing atomic, multi-writer, multi-reader registers. The simplest such implementation was presented by Vitanyi and Awerbuch in [VA86]. They implement an atomic, $(w, r)$-register, using atomic, $(1,1)$ physical registers. In this implementation the physical registers are divided into two fields: a value field and a label field. The value field stores the last logical value that was written by the its owner ${ }^{1}$, if the owner is a logical writer; if the owner is a logical reader then the value field holds the last value returned by the owner. The only operations which access the value field are copying a value to this field or from it. The label field stores all the coordination information needed for the implementation. We call implementations in which each register is divided into a value field and a label field and in which the only permitted operations accessing the value field are copy operations, label based implementations. We rename the label field to be called the coordination field and reserve the term label for the most basic coordination unit. In our implementations each coordination field consists of several labels. The complexity of a label-based implementation is measured by several criteria. The two most important criteria are:

1. Space Complexity - The maximal size of a coordination field of any physical register. (This criterion is often called label-size).

\footnotetext{
${ }^{1}$ The writer of every physical register is called its owner.
} 
2. Time Complexity - The maximal number of physical actions executed during a single logical read or write operation.

In the [VA86] implementation labels are time-stamps. This causes its main drawback, namely: unboundedness. The actual size of a label in any logical action is logarithmic in the number of write actions performed prior to that action. The time complexity of this implementation is linear in $n$, the total number of processors.

Several researchers have devised bounded label-based implementations for atomic, multi-writer, multi-reader registers, using single-writer, multi-reader physical registers: The first implementation was proposed in [VA86] and was found to be erroneous. The second implementation was proposed by Peterson and Burns in [PB87] - this implementation has a bug which was discovered and corrected by Schaffer in [Sc89]. In this implementation the space complexity is $O(w)$ and the time complexity is $O\left(w^{2}\right)$. Israeli and $\mathrm{Li}$, in [IL87], suggested bounded time-stamps as a bounded primitive to capture the temporal relationship among asynchronous processors. Using this method they devised an implementation which runs in linear time and with $O(n)$ space complexity. Other implementations with an inferior complexity are proposed by Abraham in $[\mathrm{Ab} 91 \mathrm{a}, \mathrm{Ab} 91 \mathrm{~b}]$. The work of Li, Tromp and Vitanyi in [LTV90] presents an implementation using atomic $(1,1)$-physical registers. The space complexity is $O(n)$ and the time complexity is linear. Since the implementation of [LTV90] uses inferior physical registers its complexity is superior to all aforementioned implementations.

Thus far all proposed bounded concurrent implementations have a space complexity which is linear in the number of writers, and in some cases even in the total number of processors. Some explanation for this phenomena was suggested by Israeli and Li in [IL87] who defined the class of Binary Comparison Protocols (or in short $B C P$ ) as the class of label-based implementations in which the labels of every two processors can be compared to find the most recent label among the two. They showed that the space complexity of any $B C P$ implementation is at least linear in the number of writers. Later $\mathrm{Li}$ and Vitanyi in [LV90] have pointed out that though the original [VA86] implementation is $B C P$ as well as all bounded implementations, in principal an implementation of an atomic register does not have to be $B C P$. To demonstrate this, they presented a sequential implementation (in sequential implementations the logical actions are executed sequentially, without overlapping) with $\mathrm{O}(\log w)$ space complexity. The sequential implementation of Li and Vitanyi uses the $i d$ s of the processes, or in other words, is not anonymous. For an anonymous implementation their method requires labels of size $2 \log n$, since the processor's $i d$ s are added to the labels. Later it was proven by Cori and Sopena in [CS90] that an anonymous implementation for $w$ writers should have at least $2 w-1$ distinct labels. They also devised a sequential register with exactly $2 w-1$ labels which improved the space complexity of the sequential [LV90] implementation by a constant. Recently it was proven by Tromp in [T92] that the sum of sizes of coordination fields in nonanonymous implementations is at least $n \log n$ which translates to $\log n$ size coordination fields assuming that the size of all coordination fields is the same.

These results leave an exponential gap between the lower and upper bounds on the space complexity of atomic register implementation. While the lower bounds take into account only to the combinatorial properties of keeping track of the last value (and therefore hold for sequential implementations as well), an actual concurrent implementation should also deal with concurrency problems; all existing implementations require linear space to do that. The significance of this gap is further emphasized when one takes a closer look at the unbounded implementation of Vitanyi and Awerbuch in [VA86]. For polynomial length executions the space complexity of this implementation is logarithmic, A linear space complexity is reached only in executions of exponential length. In other words: The bounded protocols supersede the unbounded protocol only in exponentially long executions. In polynomial length executions, which are often viewed as a better model for real-life situations, ensuring the theoretical boundedness requires an exponential overhead.

The natural question arising here is: Is this cost necessary? The answer is negative as we show 
by presenting two bounded, concurrent, label-based implementations for atomic, multi-writer, multireader register, with logarithmic space complexity. The first implementation uses atomic, $(1, n)$ physical registers ${ }^{2}$. The second implementation uses atomic, $(1,1)$-physical registers. These implementations are the first to break the linear space barrier for atomic multi-writer registers; moreover, by the lower bound proven by Cori and Sopena in [CS90] and Tromp in [T92], the logarithmic space complexity is optimal. The time complexity of both implementations is linear, which is obviously optimal. Both implementations are self-stabilizing: Regardless of the system's initial state, it eventually reaches a legitimate state - a state which can be reached in a legally initialized system. In such an arbitrary initial state the processors may be in arbitrary states (e.g. in the middle of some logical action) and the physical registers may hold arbitrary values.

To represent temporal relations among protocol executions we use precedence graphs. These graphs which were introduced by Israeli and $\mathrm{Li}$ in [IL87], are time-dependent graphs whose nodes and edges at any given time are determined by the labels stored in the processors' registers at that time. Label $\ell_{1}$ precedes $\ell_{2}$ if there is a directed edge $\left(\ell_{2}, \ell_{1}\right)$ in the precedence graph. Following [ILV87] we use dynamic precedence trees in which the outdegree of every node is at most 1; each label points to at most one other preceding label. At any given time the precedence graph is a forest of intrees - trees whose edges are directed towards the root. For each individual label $l b$ the set of labels whose temporal relationship with $l b$ can be found by direct comparison includes the labels whose edges point to $l b$, and the single label to which $l b$ 's edge points. Hence our protocols are indeed not $B C P$. Each path of a precedence intree is ordered temporally but labels on distinct paths are in general not comparable. The paths of any precedence intree are ordered lexicographically, by the $i d s$ of (the processors which generated) their nodes. The most preceded path in the precedence forest is called the frontal branch of the forest. Roughly speaking the frontal branch consists of nodes which are generated by recent write actions and the last node on this branch is the last serialized write action.

The rest of this paper is organized as follows: In Section 2 we formally define the model of computation and the implementation problem. In Section 3 we explain the data structure used by our protocols and present a sequential implementation. The sequential implementation serves as an exposition for the ideas which are later used in the concurrent implementations. The $(1, n)$ and the $(1,1)$ implementations are presented in Section 4 and Section 5 respectively.

\section{The MODEL}

In this section we define the model of computation and the atomic register implementation problem.

A system consists of a set of processing entities called processors, and a set of memory entities called registers. Processors access registers by executing read and write operations. Each operation is associated with a processor that can execute the operation and with a register which is accessed by the operation. Each register has a set of permitted values, a set of writer processors and a set of reader processors. A write operation to register $R E G$ is executed by some writer of $R E G$, it gets a permitted value of $R E G$ as an input parameter and stores the value in $R E G$. Analogously, a read operation from register $R E G$ is executed by some reader of $R E G$, it retrieves the (permitted) value stored in $R E G$ and returns it as an output parameter. Processors are defined by their programs whose building blocks are instructions; each instruction starts with a number of internal computations which is succeeded by at most one operation. Each program has a distinguished instruction called the program's initial instruction. Each register has a distinguished value called the register's initial value.

The execution of an operation is called an action. Under the global time model, each action has a starting time and an ending time. The time interval between the starting and ending times of action $a$ is the execution interval of $a$. Actions whose execution intervals are overlapping are called

\footnotetext{
${ }^{2}$ Since we assume that a writer always "knows" the values it writes we count it among the readers of its register.
} 
overlapping actions. Each processor is assumed to have a program counter which at any time points to the next instruction to be executed by the processor. When the system is initialized all program counters point to the processors' respective initial instructions, and each register holds its initial value. The actions of a processor are determined by its program and by the values returned by earlier read actions; since processors are serial entities the actions of a processor never overlap. Thus, an execution of processor $P$ is a sequence of non-overlapping actions of $P$, with their starting and ending times and their corresponding values.

Let $R E G$ be some register. A set $A$ of $R E G$ 's actions can be serialized, if every action $a, a \in A$, can be assigned a distinct point in time which lies within its execution interval, called the serialization time of $a$, such that the following serialization condition is satisfied:

Any read action returns the value of the write action last serialized before it.

A set of actions that access $R E G$, is an execution of $R E G$, if the set satisfies its consistency guarantee. If $R E G$ is atomic then its consistency guarantees state that each of its executions can be serialized. A sequence of actions $E$ is a system execution if for every processor $P$, the set of actions of $P$ in $E$ is an execution of $P$, and for every register $R E G$, the set of actions of $R E G$ in $E$ is an execution of $R E G$. Those executions are called the induced subexecutions of $P$ and $R E G$, respectively, under $E$. Two executions of processor $P, a_{1}, a_{2}, \ldots$ and $b_{1}, b_{2}, \ldots$ are equivalent, if for all $i, i>0 a_{i}$ and $b_{i}$ are executions of the same operation $o_{i}$. If $o_{i}$ is a write operation then the written values are equal; if $o_{i}$ is a read operation then the read values are equal; thus the only differences allowed between the two executions are in the starting and ending time of their actions. Two system executions $E_{1}$ and $E_{2}$ are equivalent if for every processor $P$ and for every register $R E G$, the induced subexecution of $P$ and $R E G$ under $E_{1}$ and under $E_{2}$ are equivalent.

An implementation of a logical register is a system whose registers are called physical registers. The operations accessing the physical registers are called physical operations. A logical operation (either read or write) is defined by a program, called protocol, whose building blocks are physical operations. The set of processors is partitioned into writers and readers. The program of (all) writers is called the writer protocol and the program of (all) readers is called the reader protocol. The writer protocol has one input parameter which is the value that should be stored in the logical register. The reader protocol should be exited through the return instruction. The return instruction does not contain any physical operation but rather returns a value which is the result of the logical read action. In label-based implementations the only instructions that access the value field of a register are copying a permitted value from this field to a local value-variable or from a local value-variable to that field. No other operations that access the value variables are allowed. In label-based implementations the input parameter to the writer protocol is not specified explicitly and is assumed to be the value corresponding to the label generated by the protocol. The return instruction returns a label whose corresponding value, which is not specified explicitly, is the returned logical value.

A logical action $a$ is an execution of a protocol. Such a logical action is a sequence of physical actions called the physical actions of $a$. The starting time of $a$ is the starting time of the first physical action of $a$. The ending time of $a$ is the ending time of the last physical action of $a$. A program is wait-free if all its executions consist of a bounded number of physical actions, where the bound may depend on the number of processors in the system. To avoid implementations which use mutual-exclusion techniques, that almost eliminate the system's concurrency, we require that the logical operations are wait-free. A logical execution is a set of logical actions of the system's processors. Every logical execution $E$ induces a corresponding physical execution which contains all the physical actions of the logical actions of $E$. An implementation is atomic if all its logical executions are serializable. An implementation is sequential if every sequential logical execution (in which no two logical actions overlap) is serializable.

In systems all of whose registers are atomic, every system execution is equivalent to (at least one) sequential execution in which every action $a$ is serialized at a distinct point in time called the occurrence 
time of $a$. When such a system implements a logical register every logical action is equivalent to a sequence of instantaneous physical actions where sequences that correspond to overlapping logical actions might be interleaved. Since every system execution is equivalent to some serial execution, any property which is correct with respect to all serial executions, is correct with respect to all executions. To prove the correctness of such an implementation, we assume that every processor continuously executes logical operations. A schedule is a sequence of processor names. Every schedule $s$ naturally induces a serial execution in which processors execute (physical) actions in the order in which they appear in $s$. Since our implementations are label-based, the execution induced by $s$ does not depend on the logical values written and read, but only on the schedule $s$. The correctness of the implementation is proven by showing that for every schedule $s$, the logical execution corresponding to the physical execution induced by $s$, is serializable.

\section{The Precedence Trees}

The three implementations presented in this paper are label-based. Temporal relations among logical actions and among the labels corresponding to these logical actions are represented by use of precedence graphs which were originally proposed by [IL87]. These are time dependent directed graphs whose nodes and edges are encoded by the labels generated during the processors' actions. The semantics of the precedence graph is: If there is an edge from label $\ell_{1}$ to $\ell_{2}$ then the action that generates $\ell_{1}$ is serialized after the action that generates $\ell_{2}$. We use dynamic precedence trees which are similar to those used in [ILV87]. In this section we describe the structure of the precedence trees used by all the implementations, and outline a simple sequential implementation of a multi-writer, multireader, register using single-writer, multi-reader registers. The sequential implementation does not improve upon the complexity of previously known sequential implementations. It is brought here as an exposition for the ideas which enable the concurrent implementations.

\subsection{Data Structure}

The writers of the implemented logical registers are denoted by $\mathcal{W}_{1}, \mathcal{W}_{2}, \ldots, \mathcal{W}_{w}$. Execution number $a$ of the writer protocol by $\mathcal{W}_{i}$ is denoted by $L_{i}^{a}$. Each individual execution of the writer protocol, $L_{i}^{a}$, generates a single label which is denoted by $\ell_{i}^{a} ; i$ and $a$ are called the $i d$ and the index of $L_{i}^{a}$ (and of $\ell_{i}^{a}$ ), respectively. Label $\ell_{i}^{a}$ is stored in the register of $\mathcal{W}_{i}$, (or in its registers in the $(1,1)$ implementation) at the end of $L_{i}^{a}$.

All our protocols share an identical structure of the labels which is described below: Each label encodes a node and a potential edge emanating from that node, where the outgoing edge is directed either towards the label itself to form a self loop, or towards a node encoded by another label. For convenience we identify the label $\ell_{i}^{a}$ with its node and denote the node by $\ell_{i}^{a}$ as well. Node $\ell_{i}^{a}$ is specified by the number $i$, which is called the $i d$ of the node, and by its address which is an integer. Since the $i d$ of all nodes of $\mathcal{W}_{i}$ is $i$, the $i d$ of a node is omitted from its encoding in $\ell_{i}^{a}$, hence the node of $\ell_{i}^{a}$ is encoded by the address field which is denoted by $\ell_{i}^{a}$.address. The edge of $\ell_{i}^{a}$ is encoded by the edge field which is denoted by $\ell_{i}^{a}$.edge. The edge field stores the $i d$ and address of the node to which the potential edge is directed, in two subfields, which are denoted by $\ell_{i}^{a}$.edge.id and $\ell_{i}^{a}$.edge.address, respectively. The potential edge emanating from $\ell_{i}^{a}$ exists in some precedence graph $G$ if for some label $\ell_{j}^{b}$ in $G$, it holds that $\ell_{i}^{a}$.edge $=\left(j, \ell_{j}^{b}\right.$.address $)$. In case this equality does not hold for any node (label) in $G$, there is no edge outgoing from $\ell_{i}^{a}$ in $G$. Our protocols make sure that in any precedence graph the aforementioned equality holds for at most one label, hence each label $\ell_{i}^{a}$ encodes at most one outgoing edge which is denoted by $e_{i}^{a}=\left(\ell_{i}^{a}, \ell_{j}^{b}\right)$, where $\ell_{j}^{b}$ is the node towards which $e_{i}^{a}$ is directed.

We require that $\ell_{i}^{a}$.edge.id $\leq i$, thus the only type of cycles that we permit are self loops. Consequently the nodes (labels) on each directed path of the precedence graph are ordered in increasing order 
of their $i d$ s from the root to the leaves. The writers' $i d$ 's lie between 1 and $w$. In case $\ell_{i}^{a} . e d g e . i d=0$ we say that $e_{i}^{a}$ exists and is directed to the virtual root label $\ell_{0}^{0}$. Since the outdegree of each node is at most 1, every precedence graph is a forest of labeled intrees - trees whose edges are directed towards the root. From now on we assume that the node set of each precedence graph includes the root label $\ell_{0}^{0}$. An edge $\left(\ell_{j}^{b}, \ell_{i}^{a}\right)$ reflects the fact that $L_{j}^{b}$ is serialized after $L_{i}^{a}$. If two edges $\left(\ell_{j}^{b}, \ell_{i}^{a}\right)$ and $\left(\ell_{k}^{c}, \ell_{i}^{a}\right)$ enter the same node $\ell_{i}^{a}$, then the serialization of actions $L_{j}^{b}$ and $L_{k}^{c}$ cannot be determined by (the precedence relation induced by) the edges of the precedence tree. In this case we serialize these actions by an ascending order of their $i d s$. (higher $i d$ s are serialized before lower $i d s$.) Later we define and use the history graph of an execution whose nodes are all labels generated during the execution. In this graph it is necessary to serialize labels with equal $i d s$ whose edges enter the same node. Since the actions of each individual processor are temporally ordered by their indices, a label with a lower index is serialized before a label with a higher index. These requirements are formally accommodated by the following lexicographic ordering of labels: Let $\ell_{i}^{a}$ and $\ell_{j}^{b}$ be two labels; $\ell_{i}^{a}$ locally precedes $\ell_{j}^{b}$ if either $j<i$ or if $i=j$ and $a<b$. Though the locally precedes relation is defined for any pair of distinct labels, it reflects a precedence relation only among labels whose edges enter the same node in the precedence graph.

Let $G$ be some precedence graph. The frontal branch of $G$ is a path which is defined recursively as follows: The first node in the frontal branch is the virtual root label $\ell_{0}^{0}$; the second node of the frontal branch of $G$ is a node whose edge enters the root and which is locally preceded by all other nodes in $G$ whose edges enter $\ell_{0}^{0}$. In general if $\alpha$ is a prefix of the frontal branch of $G$ whose last node is $\ell_{i}^{a}$ then the next node in the frontal branch is the label whose edge enters $\ell_{i}^{a}$ and which is locally preceded by all other labels whose edges enter $\ell_{i}^{a}$. The last node in the frontal branch of $G$ (whose indegree is 0 ), is called the last node of $G$. Let $e_{1}=\left(\ell_{i}^{a}, \ell_{k}^{c}\right)$ and $e_{2}=\left(\ell_{j}^{b}, \ell_{k}^{c}\right)$ be two edges in some precedence graph $G$, such that $e_{1}$ belongs to the frontal branch of $G$ (that is $\ell_{j}^{b}$ locally precedes $\ell_{i}^{a}$ ). In this case we say that $e_{1}$ excludes $e_{2}$ from the frontal branch of $G$. A pictorial description of a precedence tree appears in Figure 1, where the edges of the frontal branch appear as solid arrows and the rest of the edges appear as dotted arrows. The basic idea in all the implementations is: At any time the labels stored

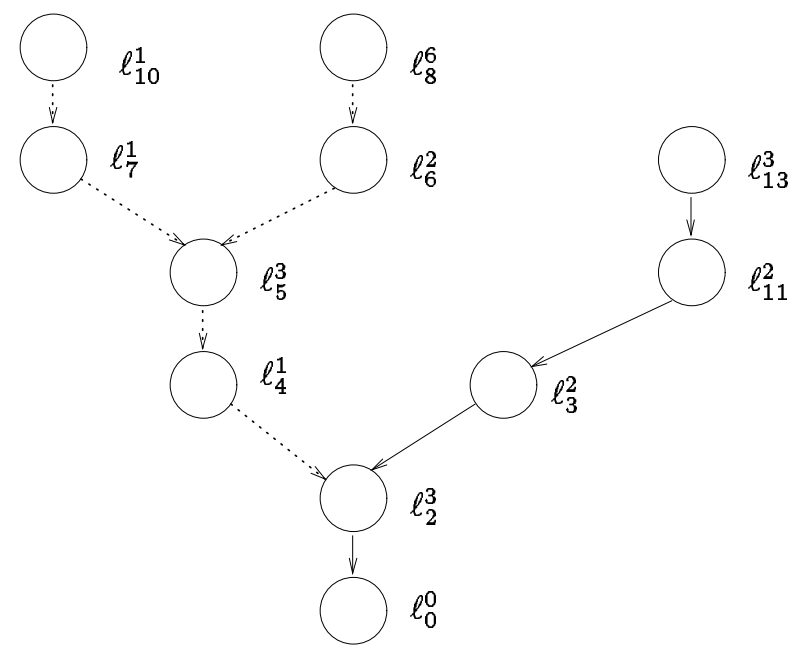

FIgURe 1. A precedence intree

in the registers encode a precedence graph whose last node belongs to the last write action completed until $t$. 


\subsection{Sequential Implementation}

In the sequential implementation each writer writes in a $(1, n)$-register called $R_{i}$, which can be read by all processors, writers and readers. (Since the implementation is sequential we need not require that the registers are atomic.) The structure of $R_{i}$ is: $R_{i}=$ (value,current). Since the value field is not used by the protocol we assume from now on that each label is written with the corresponding value and omit the values from the protocol's description. In this implementation readers do not write. The current labels at time $t$ are the labels stored in the processors' registers at time $t$. The collect operation consists of reading the labels of all writers and computing the precedence graph. This precedence graph is called the current graph, its nodes are the root and all the current labels, that is $w+1$ nodes.

Let $\alpha$ be a path in a precedence tree. The $i$-prefix of $\alpha$, denoted by $\alpha / i$, is the prefix of $\alpha$ which contains all nodes whose $i d$ is less then $i$. The protocol for $\mathcal{W}_{i}, 1 \leq i \leq w$, works as follows: $\mathcal{W}_{i}$ collects the labels of all writers, computes the frontal branch and chooses a new label which replaces its previous active label. The edge of the new label is directed towards the last node in the $i$-prefix of the frontal branch of the current graph. The new edge excludes the rest of the previous frontal branch from the new frontal branch. At the same time $\mathcal{W}_{i}$ invalidates all edges which are directed towards its previous label, by choosing a new address which is not the address of the edge field of any writer. The reader protocol is simply to collect the current graph and to return its last node. The register of $\mathcal{W}_{i}, R_{i}$, is initialized to $((i, 0), 0)$. At the initial state all edges of the current graph are self-loops and its initial frontal branch contains only the virtual root label $\ell_{0}^{0}$. The initial logical value is the value corresponding to $\ell_{0}^{0}$ which can be chosen freely from the permitted values of the logical register.

Correctness of the sequential implementation is straight forward and is left to the reader. In order to allow a writer to invalidate (at most) $w-1$ edges of the precedence tree, it should have at least $w$ possible addresses. Therefore for each writer, the size of the edge field is $2 \log w$, the size of the address field is $\log w$. The protocol's space complexity which in this case is equal to the size of one label is therefore $3 \log w$. Every execution of the writer protocol consists of $w+1$ physical actions assuming that each writer reads its own register; every execution of the reader protocol consists of $w$ actions, hence the time-complexity of the seqential implementation is $O(w)$.

\section{Multi-Writer out of Multi-Reader Registers}

In this section we present a concurrent implementation of a $(w, r)$-atomic register using physical $(1, n)$ atomic registers. In this implementation communication is again one-sided, readers do not write. An important design decision in this implementation is to serialize all logical write actions independently of the scheduling of the logical read actions. Serialization of the logical read actions is then done in accordance with the serialization of the write actions. Therefore this section is divided into two subsections: The first subsection presents the writer protocol and the serialization scheme for the logical write actions, and continues with a proof of the correctness of this serialization scheme. The second subsection presents the reader protocol and the serialization scheme for the logical read actions, and then proceeds to prove the correctness of the serialization scheme for the read actions. Together they imply the correctness of the entire implementation.

\subsection{The Writer Protocol}

\subsubsection{Description}

The writer protocol is obtained by adjusting the sequential writer protocol to the concurrent environment while keeping its basic ideas and data structure. The structure of the labels in this implementation is identical to the structure of labels in the sequential implementation and once more they 
encode a labeled intree as a precedence graph whose last node corresponds to the last completed write action. In a concurrent environment however, a writer cannot simply collect a precedence graph and replace its current label by a new label which becomes the last in the frontal branch of the collected graph. If the protocol were to work in this fashion a new label $\ell_{i}^{a}$ might be pointed at by a label of another write action which is completed at an earlier time, immediately when $\ell_{i}^{a}$ is written. In this way the precedence graph may not reflect the temporal order of actions. This problem does not rise in the sequential implementation which supports only non-overlapping logical actions. To overcome this problem the coordination field of a writer's register in this implementation consists of two labels called new and current. During $L_{i}^{a}$, the new field holds a tentative choice for $\ell_{i}^{a}$, the next label of $\mathcal{W}_{i}$. The final $\ell_{i}^{a}$ is written in the current field in the last physical action of $L_{i}^{a}$. The details of this mechanism are explained below. The structure of $R_{i}$, the register of $\mathcal{W}_{i}$ is: $R_{i}=($ new, current), and the space complexity is equal to the size of two labels rather than one.

Similar to the sequential implementation the register of $\mathcal{W}_{i}, R_{i}$, is initialized to $((i, 0), 0,(i, 0), 0)$. That is, at the initial state all edges of the current graph are self loops, its initial frontal branch contains only the root label $\ell_{0}^{0}$ and the initial logical value is the value corresponding to $\ell_{0}^{0}$, which can be chosen freely from the set of all permitted values. Let $\ell_{i}^{a}$ and $\ell_{j}^{b}$ be two labels, the fact that $\ell_{i}^{a}$ and $\ell_{j}^{b}$ are actually the same label, i.e. $i=j$ and $a=b$, is denoted by $\ell_{i}^{a} \equiv \ell_{j}^{b}$. Since the processors cannot compute the relation $\equiv$, the protocol uses the relation $\ell_{j}^{b} \simeq \ell_{j}^{c}$ which denotes the fact that $\ell_{j}^{b}$.edge $=\ell_{j}^{c}$.edge and $\ell_{j}^{b}$.address $=\ell_{j}^{c}$.address.

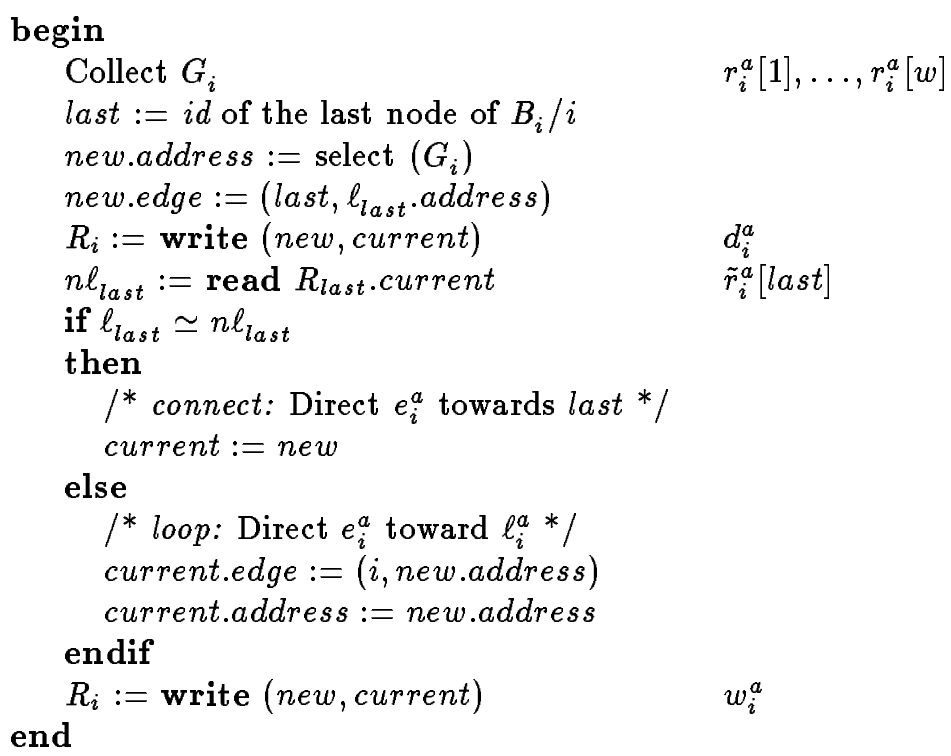

FIgURE 2. The protocol for $\mathcal{W}_{i}$ in the $(1, n)$ implementation

The writer protocol appears in Figure 2. We now describe the protocol assuming that $L_{i}^{a}$ is executed: First $\mathcal{W}_{i}$ executes the procedure collect in which it reads the registers of all other writers and computes a graph, denoted by $G_{i}^{a}$. The labels of $G_{i}^{a}$ are those read from the current fields of all registers. Execution of collect takes $w$ atomic physical actions which are denoted by $r_{i}^{a}[1] \ldots r_{i}^{a}[w]$. During collection of $G_{i}^{a}$ some writers may change their labels, therefore $G_{i}^{a}$ is not necessarily equal to the current graph at any time during its collection. The frontal branch of $G_{i}^{a}$ is denoted by $B_{i}^{a}$. The node with the maximal $i d$ in $B_{i}^{a} / i$ is the last node of $B_{i}^{a} / i$. Let $\ell_{\text {last }}$ be the last node of $B_{i}^{a} / i$. After $G_{i}^{a}$ is 
collected, $\mathcal{W}_{i}$ chooses a tentative new label whose edge is directed towards $\ell_{\text {last }}$ and whose address is obtained by the function select; this function ensures that the new label is not the head of any (existing or potential) edge encoded by any current or new label read during $L_{i}^{a}$. In this way all edges entering $\ell_{i}^{a-1}$ are invalidated. In addition the function select ensures that new.address $\neq \ell_{i}^{a-1}$.address even if no other edge is directed towards $\ell_{i}^{a-1}$; thus a writer never uses the same address twice in a row. The chosen label is declared by $\mathcal{W}_{i}$ by writing it into the new field of $R_{i}$ while the current label is not changed. The declaring write action is denoted by $d_{i}^{a}$. Following $d_{i}^{a}, \mathcal{W}_{i}$ rereads the register of $\mathcal{W}_{\text {last }}$, the writer whose label is last in $B_{i}^{a} / i$. This second read action is denoted by $\tilde{r}_{i}^{a}[$ last $]$. The label read in action $\tilde{r}_{i}^{a}[$ last $]$ is called the target label of $L_{i}^{a}$. The logical write action $L_{i}^{a}$ is concluded by a final physical write action which is denoted by $w_{i}^{a}$. In this action $\mathcal{W}_{i}$ replaces its current label as follows: Let $\ell_{\text {last }}$ and $n \ell_{\text {last }}$ be the two labels read by $\mathcal{W}_{i}$ in actions $r_{i}^{a}[$ last $]$ and $\tilde{r}_{i}^{a}[$ last $]$ respectively. If $\ell_{\text {last }} \simeq n \ell_{\text {last }}$ then new label is assigned to current, in this case we say that $L_{i}^{a}$ connects. If however $\ell_{\text {last }} \not n n \ell_{\text {last }}$ then current is chosen such that its edge is a self-loop directed towards $\ell_{i}^{a}$ itself. In this case we say that $L_{i}^{a}$ loops.

The function select considers at most $2 w-1$ addresses, thus $2 w$ possible addresses are needed in order to ensure that select can always find an unused address. Therefore, the size of the edge field in each label is is $\log w+O(1)$ and the size of the address field is also $\log w+O(1)$. The protocol's space complexity which in this case is equal to the size of two labels is therefore $O(\log w)$. Every execution of the writer protocol consists of $w+2$ physical actions assuming that each writer reads its own register; every execution of the reader protocol consists of $3 w$ actions, hence the time-complexity of this implementation is $O(w)$.

\subsubsection{Serialization Scheme for Logical Write Actions}

The atomicity of an implementation is proved by showing that for every sequential physical execution, the induced logical execution can be serialized. A sequential execution is entirely determined by its schedule; the complete asynchrony of the system means that the only points in time which can be used to serialize a logical action, are the occurrence times of the physical actions, and whenever some flexibility is possible, the intervals between these occurrence times. For this reason we sometimes use the name of an action to denote its occurrence time. Whenever we say that some property $p$ holds at action $a$ we mean that $p$ holds right after the occurrence time of $a$. In the following subsections we fix some arbitrary sequential physical execution with respect to which all definitions and proofs are being made, since this fixed execution is arbitrary, the results hold for every system execution of the implementation. The logical write actions are serialized by an explicit assignment of serialization time for each action. This assignment is done using a History graph - a precedence graph whose structure reflects the execution of the system. The history graph which is not computable by the processors plays a key-role in the correctness proof of our protocols.

Definition 1: Let $E$ be an execution of the system. Let $\tau$ be the minimal time interval between any two successive physical actions in $E$. The History graph of $E, H$, is a time dependent graph which is constructed incrementally during the execution, as follows:

1. $H^{0}$ is the History graph at time 0 (before the execution begins). It contains only the root node $-\ell_{0}^{0}$.

2. Let $L_{i}^{a}$ be an arbitrary logical write action. Let $t$ be the time when $w_{i}^{a}$ occurs, The graph $H^{t}$ is obtained from $H^{t-\tau}$ by adding the node, $\ell_{i}^{a}$, and the directed edge $e_{i}^{a}$ as follows: If $L_{i}^{a}$ connects then $e_{i}^{a}$ is directed towards the target label of $L_{i}^{a}$, otherwise ( $L_{i}^{a}$ loops) $e_{i}^{a}$ is a self-loop. According to our convention of using an action's name to denote its occurrence time, $H^{t}$ is also denoted by $H^{w_{i}^{a}}$. 
At any time $t$, the history graph $H^{t}$ is a precedence forest which consists of a single precedence tree and some disjoint self-loops. The frontal branch of $H^{t}$ is denoted by $B_{H}^{t}$. In order to serialize the logical actions we first partition the set of logical write actions into two subsets, good and bad, where a good write action is a write action whose label is last in $B_{H}^{t}$ at the time $t$ that it joins the history graph.

Definition 2: Let $L_{i}^{a}$ be an arbitrary logical write action Action. $L_{i}^{a}$ is good if $\ell_{i}^{a}$ is last in $B_{H}^{w_{i}^{a}}$. A logical write action which is not good, is bad.

Obviously if $L_{i}^{a}$ loops then it is bad, but there are many cases in which $L_{i}^{a}$ connects and it is also bad. Using the partition of logical write actions to good and bad we assign a serialization time to every logical write action. The serialization time establishes a total order on the set of logical write actions:

DEFINITION 3: Let $t$ be the occurrence time of $w_{i}^{a}$. Define the serialization time of $L_{i}^{a}$ as follows:

1. If $L_{i}^{a}$ is good, that is, $\ell_{i}^{a}$ is the last node of $B_{H}^{t}$, then $L_{i}^{a}$ is serialized at $t$.

2. If $L_{i}^{a}$ is bad and $\ell_{j}^{b}$ is the last node of $B_{H}^{t}$ then $L_{i}^{a}$ is serialized before $L_{j}^{b}$ and after any other physical action which precedes $w_{j}^{b}$. In this case we say that $L_{i}^{a}$ is serialized by $L_{j}^{b}$. In case two bad write actions, $L_{i}^{a}$ and $L_{j}^{b}$, are serialized by the same good write action $L_{k}^{c}, L_{i}^{a}$ and $L_{j}^{b}$ are serialized by an ascending order of their $i d$ s.

Let $t$ be the occurrence time of an arbitrary physical action. Under the defined serialization time the last node in $B_{H}^{t}$ is the most recent write action, that is the value of the logical register.

\subsubsection{Correctness of the Serialization Scheme for Logical Write Actions}

In the correctness proof we have to prove that the serialization time satisfies the serialization requirements for atomic registers. In Theorem 9 we prove that every logical write action is serialized within its execution interval. In Theorem 11 we prove that all graphs collected by the writers are precedence graphs. We start the correctness proof with some technical lemmas.

Lemma 1: Let $\ell_{i}^{a}$ be a node in $H^{t}$. If $\ell_{i}^{a} \notin B_{H}^{t}$, then for every $t^{\prime}>t, \ell_{i}^{a} \notin B_{H}^{t^{\prime}}$.

Proof: If $L_{i}^{a}$ loops then $\ell_{i}^{a}$ is not connected to the root at any time $t^{\prime}>t$. Assume that $L_{i}^{a}$ connects and denote the path in $H^{t}$ from $\ell_{i}^{a}$ to the root by $\alpha$. Since $\ell_{i}^{a} \notin B_{H}^{t}$, let $\left(\ell_{k}^{c}, \ell_{j}^{b}\right)$ be the first edge which is in $\alpha$ but not in $B_{H}^{t}$ and let $\left(\ell_{\ell}^{d}, \ell_{j}^{b}\right)$ be the edge excluding $\left(\ell_{k}^{c}, \ell_{j}^{b}\right)$ from $B_{H}^{t}$. No edge of $H$ is ever deleted, therefore at any time $t^{\prime}>t,\left(\ell_{\ell}^{d}, \ell_{j}^{b}\right)$ excludes the suffix of $\alpha$, and in particular $\ell_{i}^{a}$, from $B_{H}^{t^{\prime}}$.

Let $a$ and $b$ be two physical atomic actions; the fact that $a$ occurs before $b$ is denoted by $a \rightarrow b$.

LEMma 2: If $\left(\ell_{i}^{a}, \ell_{j}^{b}\right), i \neq j$, is an edge in $H$ then $w_{j}^{b}$ occurs before $w_{i}^{a}$ (i.e. $\left.w_{j}^{b} \rightarrow w_{i}^{a}\right)$.

Proof: According to the definition of the history graph, $\left(\ell_{i}^{a}, \ell_{j}^{b}\right), i \neq j$, is an edge in $H$ only if the $i d$ of the last node of $B_{i}^{a} / i$ is equal to $j$, and the label read in $\tilde{r}_{i}^{a}[$ last $]$ is $\ell_{j}^{b}$. Therefore $w_{j}^{b} \rightarrow \tilde{r}_{i}^{a}[j]$. Since $\tilde{r}_{i}^{a}[j] \rightarrow w_{i}^{a}$, we get $w_{j}^{b} \rightarrow w_{i}^{a}$. 
LEMMA 3: If $\ell_{i}^{a} \in B_{H}^{t}$ then $L_{i}^{a}$ is good.

Proof: By Lemma $1 \ell_{i}^{a} \in B_{H}^{w_{i}^{a}}$. Lemma 2 implies that for any edge $\left(\ell_{j}^{b}, \ell_{i}^{a}\right)$ in $H, w_{i}^{a} \rightarrow w_{j}^{b}$. Hence the indegree of $\ell_{i}^{a}$ in $H^{w_{i}^{a}}$ is 0 , and $\ell_{i}^{a}$ is last in $B_{H}^{w_{i}^{a}}$. By definition $L_{i}^{a}$ is good.

LEMMA 4:

(a) The sequence of node $i d s$ along any path of the history graph from the leaf towards the root is strictly decreasing.

(b) Every path of the history graph contains at most one label of every writer.

Proof: (a) According to the protocol $e_{i}^{a}$ is directed towards the last node of $B_{i}^{a} / i$. Since $B_{i}^{a} / i$ contains only nodes whose $i d<i$, the proof follows. (b) is implied immediately by (a).

Due to concurrency it is not guaranteed that the edges of a graph collected by any writer or reader belong to the history graph. A weaker relationship between the edges of the collected graphs and the edges of the history graph is depicted in the next lemma:

Lemma 5: If $\left(\ell_{i}^{a}, \ell_{j}^{b}\right)$ is an edge in $G_{k}^{c}$ then there exists an integer $r, r \geq 0$, such that $\left(\ell_{i}^{a}, \ell_{j}^{b+r}\right)$ is an edge in $H$.

Proof: The lemma holds trivially if $i=j$. Assume $i>j$, by the definition of the history graph, there exists some integer $r$ such that $\left(\ell_{i}^{a}, \ell_{j}^{b+r}\right)$ is an edge in $H$, and $\ell_{j}^{b+r}$ is the current label of $\mathcal{W}_{j}$ at $\tilde{r}_{i}^{a}[j]$. To prove the lemma we have to show that $r \geq 0$. Assume by way of contradiction that $r<0$. Since $\left(\ell_{i}^{a}, \ell_{j}^{b}\right)$ and $\left(\ell_{i}^{a}, \ell_{j}^{b+r}\right)$ are both edges of some precedence graph, it holds that $\ell_{j}^{b}$.address $=\ell_{j}^{b+r}$.address, thus if $r<0$ then $r<-1$, because a writer never uses the same address twice in a row. Therefore $w_{j}^{b+r} \rightarrow w_{j}^{b-1} \rightarrow r_{j}^{b}[i]$. Consider actions $d_{i}^{a}$ and $r_{j}^{b}[i]:$ In $d_{i}^{a}, \mathcal{W}_{i}$ declares that $e_{i}^{a}$ may be directed towards the current label of $\mathcal{W}_{j}$ read in $r_{i}^{a}[j]$, and in $r_{j}^{b}[i], \mathcal{W}_{j}$ reads the register of $\mathcal{W}_{i}$. We first show that the case $r_{j}^{b}[i] \rightarrow d_{i}^{a}$ is impossible. In this case we get that $w_{j}^{b+r} \rightarrow w_{j}^{b-1} \rightarrow r_{j}^{b}[i] \rightarrow d_{i}^{a} \rightarrow \tilde{r}_{i}^{a}[j]$, and in particular $w_{j}^{b+r} \rightarrow w_{j}^{b-1} \rightarrow \tilde{r}_{i}^{a}[j]$. Therefore $\ell_{j}^{b+r}$ is not the current label of $\mathcal{W}_{j}$ when $\tilde{r}_{i}^{a}[j]$ occurs, and the edge $\left(\ell_{i}^{a}, \ell_{j}^{b+r}\right)$ does not belong to $H$, contradiction.

The proof is completed by showing that the case $d_{i}^{a} \rightarrow r_{j}^{b}[i]$ is also impossible. First we prove that under this assumption, either the new label or the current label read by $\mathcal{W}_{j}$ in action $r_{j}^{b}[i]$, is $\ell_{i}^{a}$. By our assumption $d_{i}^{a} \rightarrow r_{j}^{b}[i]$. By the protocol $r_{j}^{b}[i] \rightarrow w_{j}^{b}$. Since $\ell_{j}^{b}$ is a node in $G_{k}^{c}$ it holds that $w_{j}^{b} \rightarrow r_{k}^{c}[j]$. Since all graphs and in particular $G_{k}^{c}$ are collected in ascending order we get that $r_{k}^{c}[j] \rightarrow r_{k}^{c}[i]$. Thus we get that $d_{i}^{a} \rightarrow r_{j}^{b}[i] \rightarrow r_{k}^{c}[i]$. Since $\ell_{i}^{a} \in G_{k}^{c}, \ell_{i}^{a}$ is the current label of $\mathcal{W}_{i}$ at $r_{k}^{c}[i]$. Therefore during the interval $\left[d_{i}^{a}, r_{k}^{c}[i]\right], \ell_{i}^{a}$ appears either as the new label of $\mathcal{W}_{i}$ or as its current label. In this case the definition of the function select implies that the new.address, selected during $L_{j}^{b}$ satisfies $\ell_{j}^{b}$.address $\neq \ell_{i}^{a}$.edge.address. On the other hand the fact that $\left(\ell_{i}^{a}, \ell_{j}^{b}\right)$ is an edge of $G_{k}^{c}$, implies that $\ell_{i}^{a}$.edge.address $=\ell_{j}^{b}$.address, a contradiction.

The fact that every label $\ell_{j}^{b} \in B_{H}^{t}$ is in $B_{i}^{a}$ and every label $\ell_{k}^{c} \in B_{i}^{a}$ is in $B_{H}^{t}$, is denoted by $B_{H}^{t} \equiv B_{i}^{a}$. In the next lemma we prove that if $\ell_{i}^{a}$ belongs to the frontal branch of the history graph at some arbitrary time $t$ then $\ell_{i}^{a}$ is the current label of $\mathcal{W}_{i}$ at $t$.

Lemma 6: If $\ell_{i}^{a} \in B_{H}^{t}$ for some time $t$, then $\ell_{i}^{a}$ is the current label of $\mathcal{W}_{i}$ at $t$. 
Proof: Assume by way of contradiction that there are labels which do not satisfy the lemma. Let $\ell_{i}^{a}$ be the label with the lowest $i d$ among these labels, that is, there exists some time $t_{0}$, such that $w_{i}^{a+1}$ occurs before $t_{0}$, while $\ell_{i}^{a} \in B_{H}^{t_{0}}$. Under these conditions Lemma 1 implies that $\ell_{i}^{a} \in B_{H}^{w_{i}^{a+1}}$. By Lemma $4(\mathrm{~b})$, the $i d s$ along any path of the history graph are distinct, therefore $\ell_{i}^{a+1} \notin B_{H}^{w_{i}^{a+1}}$. Let $\ell_{m}^{e}$ be the last node of $B_{i}^{a+1} / i$. Since $\ell_{i}^{a+1} \notin B_{H}^{w_{i}^{a+1}}, \ell_{m}^{e}$ is not the last node of $B_{H}^{w_{i}^{a+1}} / i$ and therefore $B_{H}^{w_{i}^{a+1}} / i \not \equiv B_{i}^{a+1} / i$.

Now we show that $B_{H}^{w_{i}^{a+1}} / i$ is a subgraph of $G_{i}^{a+1}$, that is, every label of $B_{H}^{w_{i}^{a+1}} / i$ is also a label of $G_{i}^{a+1}$. Let $\ell_{m}^{e}, m<i$, be an arbitrary label in $B_{H}^{w_{i}^{a+1}} / i$. Since $\ell_{i}^{a} \in B_{H}^{w_{i}^{a+1}}$, Lemma 2 implies that $w_{m}^{e} \rightarrow w_{i}^{a}$ and $\ell_{m}^{e}$ is on the path from $\ell_{i}^{a}$ to the root. Therefore, for any $t$ during the interval $\left[w_{i}^{a}, w_{i}^{a+1}\right]$ it holds that $\ell_{m}^{e} \in B_{H}^{t}$. Since $w_{i}^{a} \rightarrow r_{i}^{a+1}[m] \rightarrow w_{i}^{a+1}$ we get that $\ell_{m}^{e} \in B_{H}^{r_{i}^{a+1}}[m]$. By the minimality of $i, \ell_{m}^{e}$ is the current label of $\mathcal{W}_{m}$ at $r_{i}^{a+1}[m]$, and therefore $\ell_{m}^{e} \in G_{i}^{a+1}$.

Let $\ell_{j}^{b}, j<i$, be the last node in the common prefix of $B_{i}^{a+1} / i$ and $B_{H}^{w_{i}^{a+1}} / i$. Such a node always exists because the root, $\ell_{0}^{0}$, belongs to both branches. Now we show that $\ell_{j}^{b}$ is not last in $B_{i}^{a+1} / i$. Assume by way of contradiction, that $\ell_{j}^{b}$ is last in $B_{i}^{a+1} / i$. In this case, a node with $i d$ smaller then $i$ and with an edge directed towards $\ell_{j}^{b}$ does not exist in $G_{i}^{a+1}$. Since $B_{H}^{w_{i}^{a+1}} / i$ is a subgraph of $G_{i}^{a+1}$, there is no incoming edge to $\ell_{j}^{b}$ in $B_{H}^{w_{i}^{a+1}} / i$, that is $\ell_{j}^{b}$ is last in $B_{H}^{w_{i}^{a+1}} / i$. Since $\ell_{j}^{b}$ is the last node in the common prefix of $B_{i}^{a+1} / i$ and $B_{H}^{w_{i}^{a+1}} / i$ and since $\ell_{j}^{b}$ is last in $B_{i}^{a+1} / i$ and in $B_{H}^{w_{i}^{a+1}} / i$ we get that $B_{i}^{a+1} / i \equiv B_{H}^{w_{i}^{a+1}} / i$, a contradiction.

Since $\ell_{j}^{b}$ is not last in $B_{i}^{a+1} / i$, let $\left(\ell_{k}^{c}, \ell_{j}^{b}\right), j<k<i$, be the edge incoming to $\ell_{j}^{b}$ in $B_{i}^{a+1} / i$. Since $\ell_{j}^{b}$ is the last node in the common prefix of $B_{i}^{a+1} / i$ and $B_{H}^{w_{i}^{a+1}} / i, \ell_{k}^{c} \notin B_{H}^{w_{i}^{a+1}} / i$. Now we show that $\left(\ell_{k}^{c}, \ell_{j}^{b}\right)$ is an edge in $H_{i}^{w_{i}^{a+1}}$. By Lemma 5 there exists some $r, r \geq 0$ such that $\left(\ell_{k}^{c}, \ell_{j}^{b+r}\right)$ is an edge in $H_{i}^{w_{i}^{a+1}}$. Since $\ell_{j}^{b} \in B_{H}^{w_{i}^{a+1}}$, the minimality of $i$ implies that $\ell_{j}^{b}$ is the current label of $\mathcal{W}_{j}$ at $w_{i}^{a+1}$. Therefore $r=0$, which implies that $\left(\ell_{k}^{c}, \ell_{j}^{b}\right)$ is an edge in $H_{i}^{w_{i}^{a+1}}$ (but not in $B_{H}^{w_{i}^{a+1}}$ ). Since $\left(\ell_{k}^{c}, \ell_{j}^{b}\right)$ is not in $B_{H}^{w_{i}^{a+1}}$, let $\left(\ell_{\ell}^{d}, \ell_{j}^{b}\right), \ell<i$, be the edge excluding $\left(\ell_{k}^{c}, \ell_{j}^{b}\right)$ from $B_{H}^{w_{i}^{a+1}} / i$. By the definition of $B_{H}^{t}, \ell_{k}^{c}$ locally precedes $\ell_{\ell}^{d}$. Since $B_{H}^{w_{i}^{a+1}} / i$ is a subgraph of $G_{i}^{a+1},\left(\ell_{\ell}^{d}, \ell_{j}^{b}\right)$ is an edge in $G_{i}^{a+1}$. Since $\ell_{k}^{c}$ locally precedes $\ell_{\ell}^{d}$ we get that $\left(\ell_{\ell}^{d}, \ell_{j}^{b}\right)$ excludes $\left(\ell_{k}^{c}, \ell_{j}^{b}\right)$ from $B_{i}^{a+1}$, contradiction to the assumption that $\left(\ell_{k}^{c}, \ell_{j}^{b}\right)$ is an edge in $B_{i}^{a+1} / i$. The lemma follows.

The next corollary forms a tighter relationship between edges of $G$ whose head belong to the frontal branch of the history graph and the corresponding edges of $H$.

CoRollaRY 7: Let $\left(\ell_{i}^{a}, \ell_{j}^{b}\right)$ be an edge in $G_{k}^{c}$. If for some $t$ after $r_{k}^{c}[i], \ell_{j}^{b} \in B_{H}^{t}$ then $\left(\ell_{i}^{a}, \ell_{j}^{b}\right)$ is an edge in $H^{t}$.

Proof: Since $\left(\ell_{i}^{a}, \ell_{j}^{b}\right)$ is an edge in $G_{k}^{c}$, Lemma 5 implies that for some $r, r \geq 0,\left(\ell_{i}^{a}, \ell_{j}^{b+r}\right)$ is an edge in $H^{t}$. Since $\ell_{j}^{b} \in B_{H}^{t}$, Lemma 6 implies that $\ell_{j}^{b}$ is the current label of $\mathcal{W}_{j}$ at $t$, therefore $r=0$.

In the next lemma and in the theorem that follows, we prove that the serialization time of each logical write action lies within its execution interval.

LEMMA 8: Let $t_{0}$ be the occurrence time of $r_{i}^{a}[j]$. If for some $t, t \geq t_{0}, B_{H}^{t} /(j+1) \not \equiv B_{i}^{a} /(j+1)$, then there exists a good logical action $L_{k}^{c}$, for some $k \leq j$, such that $w_{k}^{c}$ occurs within the time interval starting at $r_{i}^{a}[k]$ and ending at $t$. 
Proof: By the assumption of the lemma, $B_{H}^{t} /(j+1) \not \equiv B_{i}^{a} /(j+1)$. Let $\ell_{\ell}^{d}, \ell \leq j$, be the node with the maximal $i d$ in the common prefix of $B_{H}^{t} /(j+1)$ and $B_{i}^{a} /(j+1)$. First we show that $\ell_{\ell}^{d}$ is not the last node of $B_{H}^{t} /(j+1)$. If $\ell_{\ell}^{d}$ is the last node of $B_{H}^{t} /(j+1)$ then since $B_{i}^{a} /(j+1) \not \equiv B_{H}^{t} /(j+1)$, $\ell_{\ell}^{d}$ is not the last node of $B_{i}^{a} /(j+1)$. Let $e_{1}=\left(\ell_{m}^{e}, \ell_{\ell}^{d}\right), m<j+1$, be the edge entering $\ell_{\ell}^{d}$ in $B_{i}^{a} /(j+1)$. Since $\ell_{\ell}^{d} \in B_{H}^{t}$, Corollary 7 implies that $\left(\ell_{m}^{e}, \ell_{\ell}^{d}\right)$ is an edge of $H^{t}$; since $m<j+1$, either $e_{1} \in B_{H}^{t} /(j+1)$ or there exists some edge $e_{2} \in B_{H}^{t} /(j+1)$ excluding $e_{1}$ from $B_{H}^{t} /(j+1)$, a contradiction to the assumption that $\ell_{\ell}^{d}$ is the last node of $B_{H}^{t} /(j+1)$.

Since $\ell_{\ell}^{d}$ is not the last node of $B_{H}^{t} /(j+1)$, let $\left(\ell_{k}^{c}, \ell_{\ell}^{d}\right), \ell<k \leq j$, be the edge entering $\ell_{\ell}^{d}$ in $B_{H}^{t} /(j+1)$. We now show that $L_{k}^{c}$ satisfies the requirements of the lemma; since $\ell_{k}^{c} \in B_{H}^{t}$ Lemma 3 implies that $L_{k}^{c}$ is good. Since $\ell_{k}^{c} \in H^{t}$, it is obvious that $w_{k}^{c}$ occurs before $t$. To complete the proof it remains to show that $r_{i}^{a}[k] \rightarrow w_{k}^{c}$. Assume by way of contradiction that $w_{k}^{c} \rightarrow r_{i}^{a}[k]$. We reach a contradiction as follows (see Figure 3): First we show that $\left(\ell_{k}^{c}, \ell_{\ell}^{d}\right)$ is an edge in $G_{i}^{a}$ (but not in
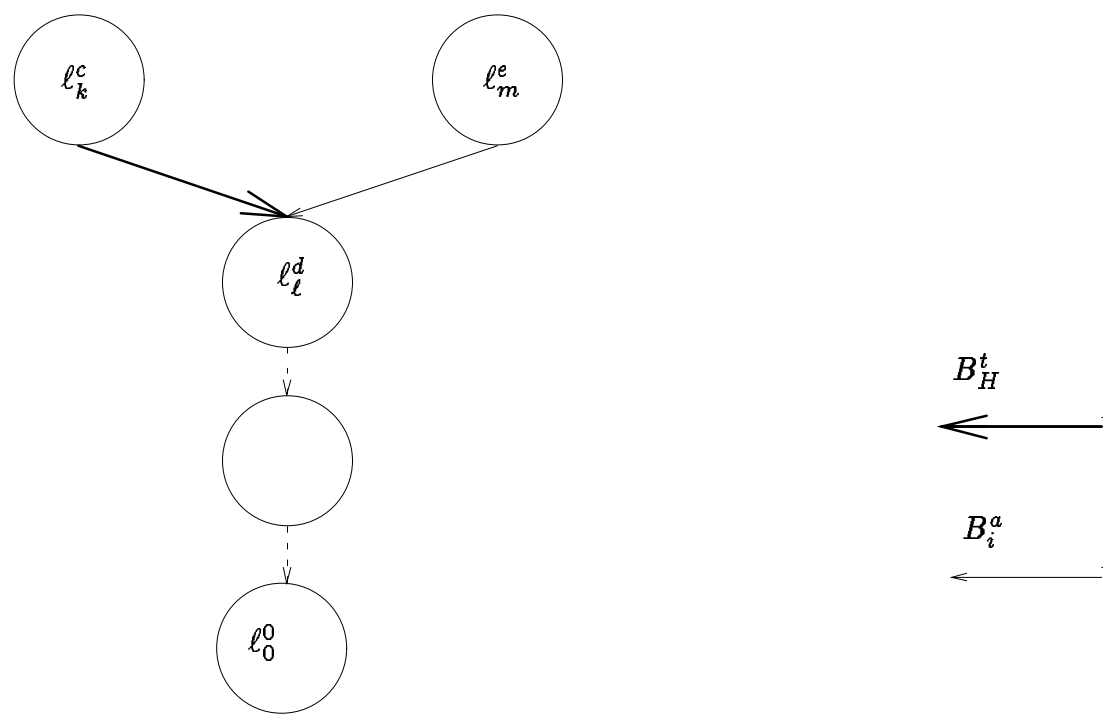

$B_{i}^{a}$

FiguRE 3. The graph for Lemma 8

$\left.B_{i}^{a} /(j+1)\right)$. By its definition, $\ell_{k}^{c}$ is a node of $B_{H}^{t}$, therefore by Lemma $6, \ell_{k}^{c}$ is the current label of $\mathcal{W}_{k}$ at $t$. By our assumption $w_{k}^{c} \rightarrow r_{i}^{a}[k]$, hence $\ell_{k}^{c}$ is a node of $G_{i}^{a}$. Therefore $\left(\ell_{k}^{c}, \ell_{\ell}^{d}\right)$ is an edge in $G_{i}^{a}$. Since $k>\ell$, and $\ell_{\ell}^{d}$ is the maximal common node in $B_{H}^{t} /(j+1)$ and $B_{i}^{a} /(j+1)$ we conclude that $\ell_{k}^{c} \notin B_{i}^{a} /(j+1)$. Let $\left(\ell_{m}^{e}, \ell_{\ell}^{d}\right), \ell<m<k$, be the edge excluding $\left(\ell_{k}^{c}, \ell_{\ell}^{d}\right)$ from $B_{i}^{a} /(j+1)$. By Corollary $7,\left(\ell_{m}^{e}, \ell_{\ell}^{d}\right)$ is an edge in $H^{t}$. Since $m<k,\left(\ell_{m}^{e}, \ell_{\ell}^{d}\right)$ excludes $\left(\ell_{k}^{c}, \ell_{\ell}^{d}\right)$ from $B_{H}^{t}$, a contradiction to the assumption that $\left(\ell_{k}^{c}, \ell_{\ell}^{d}\right)$ is in $B_{H}^{t} /(j+1)$. The lemma follows.

Theorem 9: Every logical write action is serialized within its execution interval.

Proof: Let $L_{i}^{a}$ be some logical write action. If $L_{i}^{a}$ is good then it is serialized at its concluding physical write $w_{i}^{a}$, which is within its execution interval. For the rest of the proof we assume that $L_{i}^{a}$ is bad. Let $\ell_{j}^{b}$ be the last node of $B_{H}^{w_{i}^{a}}$. According to the serialization definition for bad write actions, $L_{i}^{a}$ is serialized by $L_{j}^{b}$. We have to prove that $L_{j}^{b}$ is serialized within the execution interval 
of $L_{i}^{a}$, that is $r_{i}^{a}[1] \rightarrow w_{j}^{b} \rightarrow w_{i}^{a}$. Since $\ell_{j}^{b} \in H^{w_{i}^{a}}$, it is clear that $w_{j}^{b} \rightarrow w_{i}^{a}$. We now prove that $r_{i}^{a}[1] \rightarrow w_{j}^{b}$. Action $L_{i}^{a}$ is bad, therefore $B_{H}^{w_{i}^{a}} / i \not \equiv B_{i}^{a} / i$. By Lemma 8 there exists a good logical action $L_{k}^{c}, k<i$, such that $w_{k}^{c}$ occurs within the time interval starting at $r_{i}^{a}[k]$ and ending at $w_{i}^{a}$. Therefore $r_{i}^{a}[k] \rightarrow w_{k}^{c} \rightarrow w_{i}^{a}$, which implies $r_{i}^{a}[1] \rightarrow w_{k}^{c} \rightarrow w_{i}^{a}$. If $L_{j}^{b} \equiv L_{k}^{c}$ then the the proof follows. Otherwise, since $L_{j}^{b}$ and $L_{k}^{c}$ are both good, and since $\ell_{j}^{b}$ is the last node in $B_{H}^{w_{i}^{a}}$, we get that $w_{k}^{c} \rightarrow w_{j}^{b}$. Therefore, $r_{i}^{a}[1] \rightarrow w_{k}^{c} \rightarrow w_{j}^{b}$, which implies $r_{i}^{a}[1] \rightarrow w_{j}^{b}$.

The fact that $L_{i}^{a}$ is serialized before $L_{j}^{b}$ is denoted by $L_{i}^{a} \Rightarrow L_{j}^{b}$. The next lemma proves that the history graph is a precedence graph with respect to the relation $\Rightarrow$. Since the history graph is not computable by the processors, the significance of this lemma is reflected in the theorem that follows in which it is proved that the graphs collected by the processors are also precedence graphs with respect to the relation $\Rightarrow$.

LEMma 10: If $\left(\ell_{i}^{a}, \ell_{j}^{b}\right), i>j$, is an edge in $H$ then $L_{j}^{b} \Rightarrow L_{i}^{a}$.

Proof: By Lemma $2 w_{j}^{b} \rightarrow w_{i}^{a}$. Consider the following cases:

Case 1: $L_{i}^{a}$ is good. In this case $L_{i}^{a}$ is serialized at $w_{i}^{a}$. Since $L_{j}^{b}$ is not serialized after $w_{j}^{b}$, and since $w_{j}^{b} \rightarrow w_{i}^{a}$ it holds that $L_{j}^{b} \Rightarrow L_{i}^{a}$.

Case 2: $L_{i}^{a}$ and $L_{j}^{b}$ are both bad. Let $\ell_{k}^{c}$ and $\ell_{\ell}^{d}$ be the last nodes in $B_{H}^{w_{i}^{a}}$ and $B_{H}^{w_{j}^{b}}$ respectively. In this case $L_{i}^{a}$ is serialized by $L_{k}^{c}$ and $L_{j}^{b}$ is serialized by $L_{\ell}^{d}$. If $\ell_{k}^{c} \equiv \ell_{\ell}^{d}$, then by Definition 3 , $L_{i}^{a}$ and $L_{j}^{b}$ are serialized by their $i d s$. Since $i>j L_{j}^{b} \Rightarrow L_{i}^{a}$. Assume $\ell_{k}^{c} \not \equiv \ell_{\ell}^{d}$. By definition $3, L_{\ell}^{d}$ is the last good write action serialized before $w_{j}^{b}$ and $L_{k}^{c}$ is the last good write action serialized before $w_{i}^{a}$. Since $w_{j}^{b} \rightarrow w_{i}^{a}$ it holds that $w_{\ell}^{d} \rightarrow w_{k}^{c}$ and therefore $L_{\ell}^{d} \Rightarrow L_{k}^{c}$. Since $L_{j}^{b}$ is serialized by $L_{\ell}^{d}$ and $L_{i}^{a}$ is serialized by $L_{k}^{c}$, we get $L_{j}^{b} \Rightarrow L_{i}^{a}$.

Case 3: $L_{i}^{a}$ is bad, $L_{j}^{b}$ is good. In this case $\ell_{j}^{b}$ is not the last node of $B_{H}^{w_{i}^{a}}$. Let $\ell_{k}^{c}$ be the last node of $B_{H}^{w_{i}^{a}}$, that is $w_{j}^{b} \rightarrow w_{k}^{c} \rightarrow w_{i}^{a}$. Since $L_{j}^{b}$ and $L_{k}^{c}$ are both good, and since $w_{j}^{b} \rightarrow w_{k}^{c}, L_{j}^{b} \Rightarrow L_{k}^{c}$. By Definition $3(2) L_{i}^{a}$ is serialized by $L_{k}^{c}$, therefore we get $L_{j}^{b} \Rightarrow L_{i}^{a}$.

TheOREM 11: If $\left(\ell_{i}^{a}, \ell_{j}^{b}\right), i>j$, is an edge in $G_{k}^{c}$ then $L_{j}^{b} \Rightarrow L_{i}^{a}$.

Proof: By Lemma 5, there exists some $r, r \geq 0$, such that $\left(\ell_{i}^{a}, \ell_{j}^{b+r}\right)$ an edge in $H$. By Lemma 10 $L_{j}^{b+r} \Rightarrow L_{i}^{a}$. If $r=0$ then we get $L_{j}^{b} \Rightarrow L_{i}^{a}$. If $r>0$ then since $L_{j}^{b} \Rightarrow L_{j}^{b+r}$ we get again that $L_{j}^{b} \Rightarrow L_{i}^{a}$.

\subsection{The Reader Protocol}

\subsubsection{Description}

Like the writer protocol, the reader protocol is obtained by adjusting the sequential reader protocol to the concurrent environment. Though the basic idea in this implementation is to keep a precedence graph whose last node is the last value written to the logical register, it is not possible to just collect a precedence graph and return the last node in its frontal branch: Due to concurrency, the current graph 
and its last node may change during the execution of the collect procedure by the reader. In particular a label of an action which should not be returned by a reader, may appear as last in its collected graph. This happens when some concurrent write actions cause the reader to see some branches of the graph as "hanging in the air". Therefore a single collection is not sufficient. Our protocol collects three precedence graphs and analyzes the differences among them to determine the returned label. The three graphs are denoted by $G, \overleftarrow{G}$, and $\tilde{G}$. Graph $\stackrel{\leftarrow}{G}$ is collected in reverse order - from $R_{w}$ down to $R_{1}$, therefore most of the lemmas proven in the previous section do not hold for $\overleftarrow{G}$. The analysis of the three graphs does not yield an accurate description of the current graph, but rather enables the reader to identify a label which satisfies the requirements for the reader protocol as follows: The identified label is either last in the history graph when the logical read action starts, and hence it is the last logical write action that is serialized before the read action starts, or the identified label is generated by a logical write action serialized within the execution interval of the logical read action. In this case the logical read is serialized immediately after the logical write. The physical actions, executed by $\mathcal{R}_{u}$, during the reader protocol are denoted by: $r_{u}[1] \ldots r_{u}[w]$, in which $G$ is collected, $\overleftarrow{r_{u}}[w] \ldots \overleftarrow{r_{u}}[1]$, in which $\stackrel{\leftarrow}{G}$ is collected, and $\tilde{r}_{u}[1] \ldots \tilde{r}_{u}[w]$, in which $\tilde{G}$ is collected. The notation $B_{i}^{a} \subset B_{j}^{b}$ is used when for each $\ell_{k}^{c} \in B_{i}^{a}$, there is a label $\ell_{k}^{d} \in B_{j}^{b}$ such that $\ell_{k}^{c} \simeq \ell_{k}^{d}$. The notation $B_{i}^{a} \simeq B_{j}^{b}$ is used when $B_{i}^{a} \subset B_{j}^{b}$ and $B_{j}^{b} \subset B_{i}^{a}$. The code of the reader protocol appears in Figure 4 .

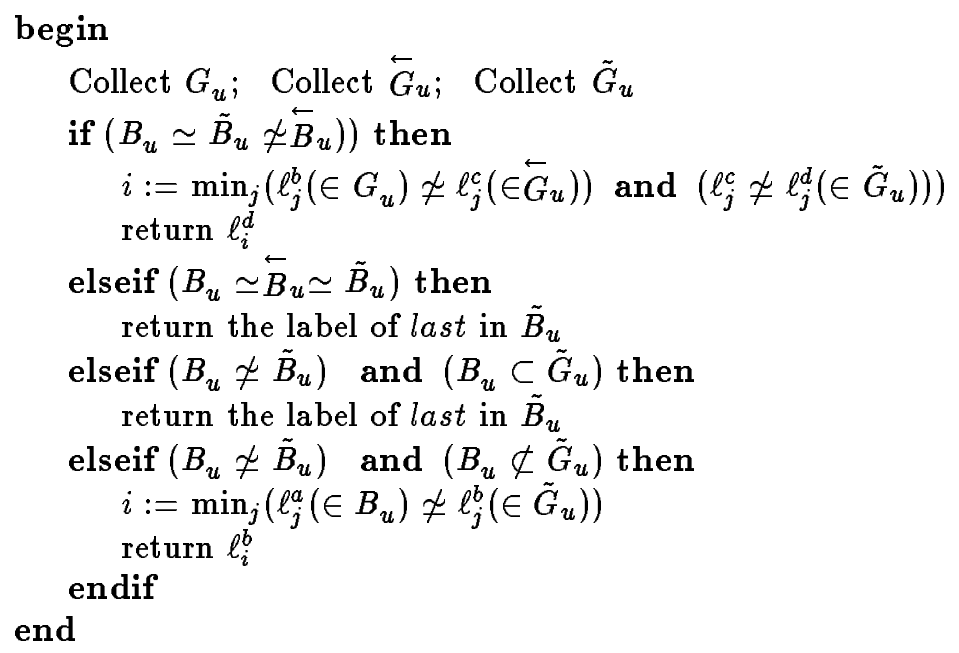

FIGURE 4. The protocol for $\mathcal{R}_{u}$ in the $(1, n)$ implementation

\subsubsection{Serialization Scheme for Logical Read Actions}

Throughout this paper $S_{u}^{a}$ denotes the $a$-th execution of the read protocol by $\mathcal{R}_{u}$. The serialization time of any logical read action is determined by the serialization time of the logical write action whose value is returned by the read action, according to the following proposition:

Proposition 12: If for any logical read action $S_{u}^{a}$ the returned label $\ell_{j}^{b}$ satisfies one of the following conditions:

1. $L_{j}^{b}$ is the logical write action that is serialized last before $S_{u}^{a}$ starts. 
2. $L_{j}^{b}$ is serialized within the execution interval of $S_{u}^{a}$.

then the implementation is atomic.

To prove the proposition correct we have to show that if for every action in some execution $E$, one of these conditions holds, then the $E$ is serializable. This is proven by the following serialization scheme:

DEFinition 4: Let $\ell_{i}^{b}$ be the label returned by $S_{u}^{a}$. Denote by $t_{s}$ and $t_{e}$ the occurrence time of $r_{u}^{a}[1]$ and $\tilde{r}_{u}^{a}[w]$ respectively. The serialization time of $S_{u}^{a}$ is defined as follows:

1. If $L_{i}^{b}$ is not serialized within the execution interval of $S_{u}^{a}$ then $S_{u}^{a}$ is serialized at $t_{s}$.

2. If $L_{i}^{b}$ is serialized within the execution interval of $S_{u}^{a}$ then $S_{u}^{a}$ is serialized by $L_{i}^{b}$, that is after $L_{i}^{b}$ and before any physical action which occurs or any logical write which is serialized after $L_{i}^{b}$. In case two logical actions are serialized by the same logical write action they are serialized by an ascending order of their ids.

\subsubsection{Correctness of the Implementation}

The correctness of the serialization scheme for logical read actions, and the correctness of the entire implementation, is based on Proposition 12 and on the following theorem:

Theorem 13: Let $\left(t_{s}, t_{e}\right)$ be the execution interval of $S_{u}$, let $\ell$ be the label returned by $S_{u}$, and let $L$ be the logical write action that produced the label $\ell$. The label $L$ satisfies one of the following two claims:

1. $L$ is the last logical write action serialized before $t_{s}$ ( and hence $\ell$ is last in $B_{H}^{t_{s}}$ ), or

2. $L$ is serialized within the interval $\left(t_{s}, t_{e}\right)$.

Proof: $\quad$ Since $\ell$ is read during the execution of $S_{u}$, it is clear that $L$ terminates before $t_{e}$. Therefore, to conclude that (2) holds, it suffices to show that $L$ is serialized after $t_{s}$. Consider the following cases (which follow the cases of the protocol):

Case 1: $B_{u} \simeq \tilde{B}_{u} \not \overleftarrow{B}_{u}$

Let $i$ be the smallest $i d$ such that $\ell_{i}^{b} \simeq \ell_{i}^{d} \not \ell_{i}^{c}$, where $\ell_{i}^{b}, \ell_{i}^{d}$ and $\ell_{i}^{c}$ are nodes in $G_{u}, \tilde{G}_{u}$, and $\overleftarrow{G}_{u}$ respectively. According to the protocol $S_{u}$ returns $\ell_{i}^{d}$. Since $\ell_{i}^{b} \not \ell_{i}^{c}$ we get that

$$
r_{u}[1] \rightarrow r_{u}[i] \rightarrow w_{i}^{c}
$$

Since $\ell_{i}^{c} \not \subset \ell_{i}^{d}$ we get that

$$
w_{i}^{c} \rightarrow r_{i}^{d}[1]
$$

Therefore from (1) and (2) we get that $r_{u}[1] \rightarrow r_{i}^{d}[1]$. Hence $L_{i}^{d}$ is serialized after $t_{s}$.

Case 2: $B_{u} \simeq \tilde{B}_{u} \simeq \overleftarrow{B}_{u}$

Let $\ell_{i}^{a}$ be the last node of $\tilde{B}_{u}$. According to the protocol $S_{u}$ returns $\ell_{i}^{a}$. Consider the following cases: 
Case 2.1: $B_{u} \not \equiv \tilde{B}_{u}$

In this case there exist two distinct labels $\ell_{j}^{b}$ and $\ell_{j}^{c}(b<c)$ of the same writer, $\mathcal{W}_{j}$, such that $\ell_{j}^{b} \in B_{u}$ while $\ell_{j}^{c} \in \tilde{B}_{u}$. Since $B_{u} \simeq \tilde{B}_{u} \ell_{j}^{b} \simeq \ell_{j}^{c}$, and in particular $\ell_{j}^{b}$.address $=\ell_{j}^{c}$.address. A writer does not use the same address twice in a row, hence $(b+1)<c$. Therefore $r_{u}[j] \rightarrow w_{j}^{b+1} \rightarrow r_{j}^{c}[1] \rightarrow w_{j}^{c}$, which implies that $L_{j}^{c}$ is serialized after $t_{s}$. If $\ell_{i}^{a} \not \equiv \ell_{j}^{c}$ then there is a path from $\ell_{i}^{a}$ to $\ell_{j}^{c}$ in $\tilde{G}_{u}$ (because $\ell_{i}^{a}$ and $\ell_{j}^{c}$ are both in $\tilde{B}_{u}$, and $\ell_{i}^{a}$ is last in $\tilde{B}_{u}$ ), hence by Theorem $11 L_{j}^{c} \Rightarrow L_{i}^{a}$. Therefore $L_{i}^{a}$ is serialized after $t_{s}$.

Case 2.2: $B_{u} \equiv \tilde{B}_{u}$

We first show that $B_{u}$ is a branch in $H^{r_{u}[w]}$. Let $\left(\ell_{j}^{b}, \ell_{k}^{c}\right)$ be an arbitrary edge in $B_{u}$. By Lemma 5 there exists an integer $r, r \geq 0$, such that $\left(\ell_{j}^{b}, \ell_{k}^{c+r}\right)$ is an edge in $H^{r_{u}[w]}$. Since $B_{u} \equiv \tilde{B}_{u}, \ell_{k}^{c}$ is a label in $\tilde{G}_{u}$, hence it is the current label of $\mathcal{W}_{k}$ at $\tilde{r}_{u}[k]$, and therefore $r=0$. Thus every edge in $B_{u}$ belongs to $H^{r_{u}}[w]$. Similarly, the assumptions $B_{u} \simeq \overleftarrow{B}_{u}$ (case 2) and $B_{u} \equiv \tilde{B}_{u}$ (case 2.2) imply that $B_{u} \equiv \overleftarrow{B}_{u} \equiv \tilde{B}_{u}$. Since $B_{u} \equiv B_{H}^{r_{u}[w]}$ (which is proven in Claim 1) we get that $\ell_{i}^{a}$ is last in $B_{H}^{r_{r}[w]}$, which implies that either $\ell_{i}^{a}$ is last in $B_{H}^{t_{s}}$ or $\ell_{i}^{a}$ is serialized after $t_{s}$.

CLAIM 1: $B_{u} \equiv B_{H}^{r_{u}[w]}$.

Proof of claim: Assume by way of contradiction that $B_{u} \not \equiv B_{H}^{r_{u}[w]}$. In this case at every time $t$ after $r_{u}[w]$ there exists some edge in $H^{t}$ that excludes a suffix of $B_{u}$ from $B_{H}^{t}$. Let $S=\overleftarrow{t}_{w}<\overleftarrow{t}_{w-1}<\ldots<\overleftarrow{t}_{1}$ be the sequence of occurrence times of actions $\overleftarrow{r}_{u}[w]$, $\overleftarrow{r_{u}}[w-1] \ldots \overleftarrow{r_{u}}[1]$ respectively. Define the function $E X(t)$ on $S$ as follows: The value of $E X(t)$ is $k$ where $k$ is the $i d$ of the label in $B_{H}^{t}$ whose edge excludes a suffix of $B_{u}$ from $B_{H}^{t}$. Note that $E X\left(\overleftarrow{t}_{w}\right)<w$ and $E X\left(\overleftarrow{t}_{1}\right)>1$. We now show that $E X$ is not increasing in $t$. Assume that $E X(t)=k, E X\left(t^{\prime}\right)=\ell$, and $t<t^{\prime}$. Let $\alpha$ denote the common prefix of $B_{H}^{t}$ and $B_{u}$, and let $\beta$ denote the common prefix of $B_{H}^{t^{\prime}}$ and $B_{u}$. Since $t<t^{\prime}, \beta$ is a (not necessarily proper) prefix of $\alpha$. If $\alpha \equiv \beta$ then $\ell \leq k$ and we are done. If $\beta$ is a proper prefix of $\alpha$ then let $\left(\ell_{j}^{b}, \ell_{i}^{a}\right)$ and $\left(\ell_{\ell}^{d}, \ell_{i}^{a}\right)$ be the edges of $B_{u}$ and $B_{H}^{t^{\prime}}$ respectively where $\left(\ell_{\ell}^{d}, \ell_{i}^{a}\right)$ excludes $\left(\ell_{j}^{b}, \ell_{i}^{a}\right)$ from $B_{H}^{t^{\prime}}$. Obviously $\ell<j<k$.

We now use the function $E X$ to reach a contradiction by showing that under these assumptions $B_{u} \not \equiv \overleftarrow{B}_{u}$. Since $\overleftarrow{G}_{u}$ is collected in reverse order (from $w$ to 1 ) and $E X$ is not increasing, there exists a time $\overleftarrow{t}_{k}, \overleftarrow{t}_{k} \in S$, such that $E X\left(\overleftarrow{t}_{k}\right)=k$. Let $\left(\ell_{k}^{b}, \ell_{j}^{c}\right)$ be the edge

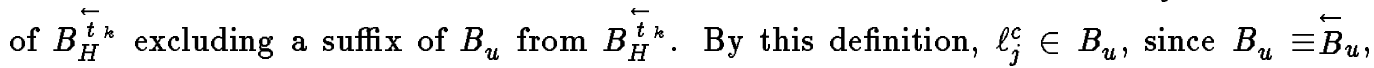
$\ell_{j}^{c} \in \overleftarrow{B}_{u}$, hence obviously $\ell_{j}^{c} \in \overleftarrow{G} u$. Since $\ell_{k}^{b}$ belongs to ${\stackrel{\leftarrow}{\boldsymbol{t}_{H}}}_{k}$, Lemma 6 implies that it is the current label of $\mathcal{W}_{k}$ at $\overleftarrow{t}_{k}$ and therefore $\ell_{k}^{b}$ is a node in $\overleftarrow{G}_{u}$. Since $\ell_{k}^{b} \in \overleftarrow{G}_{u}$ and $\ell_{j}^{c} \in \overleftarrow{G}_{u}$, we get that $\left(\ell_{k}^{b}, \ell_{j}^{c}\right)$ is an edge in $\overleftarrow{G}_{u}$. Since $\left(\ell_{k}^{b}, \ell_{j}^{c}\right)$ excludes a suffix of $B_{u}$ from $\overleftarrow{B}_{H}^{\overleftarrow{t}_{k}},\left(\ell_{k}^{b}, \ell_{j}^{c}\right)$ excludes a suffix of $B_{u}$ from $\overleftarrow{B}_{u}$, contradiction to the assumption that $B_{u} \equiv \overleftarrow{B}_{u}$

Case 3: $B_{u} \not \tilde{B}_{u}$ and $B_{u} \subset \tilde{G}_{u}$.

Let $\ell_{i}^{a}$ be the last node of $\tilde{B}_{u}$. According to the protocol $S_{u}$ returns $\ell_{i}^{a}$. In the sequel we show that $L_{i}^{a}$ is serialized after $t_{s}$. Since $B_{u} \subset \tilde{G}_{u}$ and $B_{u} \not \tilde{B}_{u}$ there is a suffix of $\tilde{B}_{u}$, none of whose labels are in $B_{u}$. Let $j$ be the $i d$ of the minimal label $\ell_{j}^{c}$ in that suffix (see Figure 5), and let $\ell_{j}^{b}$ be the label of $\mathcal{W}_{j}$ in $G_{u}$. By this definition $\ell_{j}^{b} \not \ell_{j}^{c}$.

In order to show that $L_{i}^{a}$ is serialized after $t_{s}$ it is enough to show that $L_{j}^{c}$ is serialized after $t_{s}$, since if $L_{i}^{a} \not \equiv L_{j}^{c}$ then there is a path from $\ell_{i}^{a}$ to $\ell_{j}^{c}$ in $\tilde{B}_{u}$, and therefore Theorem 11 implies 


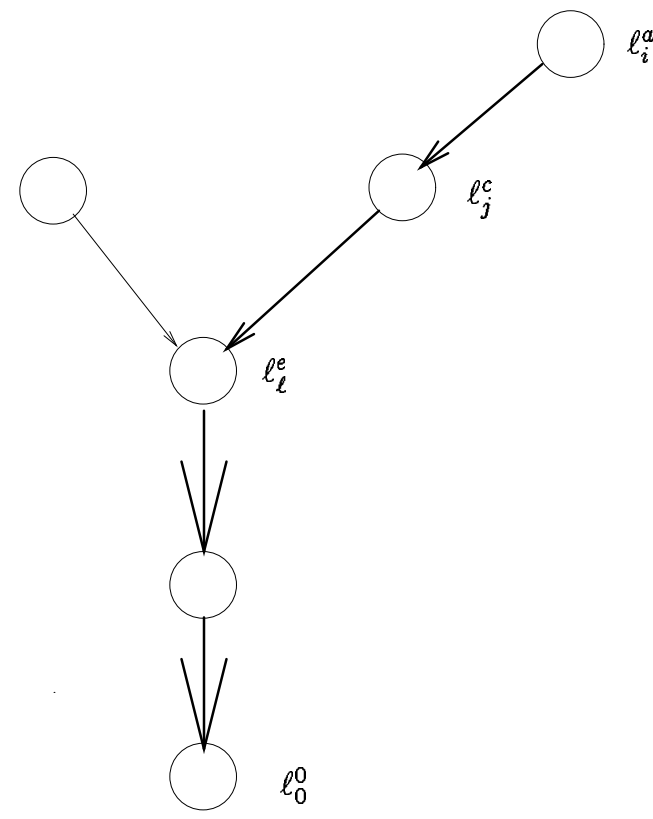

Case A

$B_{u}$ is not a prefix of $\tilde{B}_{u}$

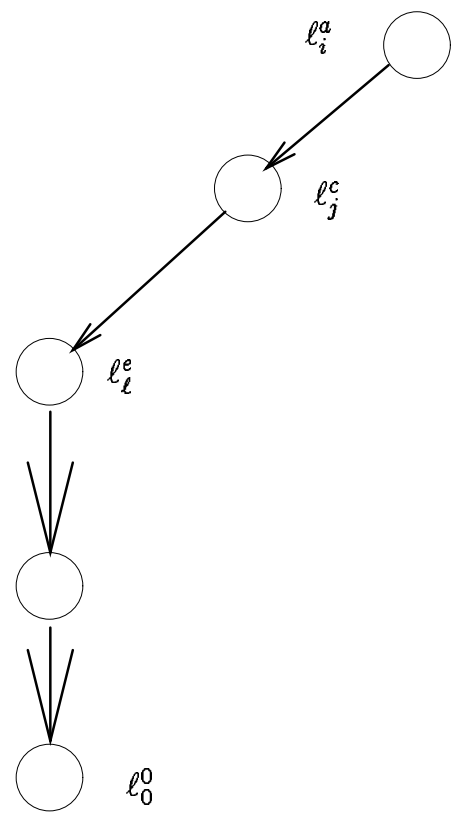

Case B $B_{u}$ is a prefix of $\tilde{B}_{u}$

Edges belong both to $B_{u}$ and $\tilde{B}_{u}$ Edges belong to $\tilde{B}_{u}$

Edges belong to $B_{u}$

FIGURE 5. Case 3 - Relation between $B_{u}$ and $\tilde{B}_{u}$ when $B_{u} \subset \tilde{G}_{u}$

that $L_{j}^{c} \Rightarrow L_{i}^{a}$. We proceed to show that $L_{j}^{c}$ is serialized after $t_{s}$ : Since $\ell_{j}^{b} \not \ell_{j}^{c}$ it is clear that $r_{u}[j] \rightarrow w_{j}^{c}$ and therefore $w_{j}^{c}$ occurs after $t_{s}$. If $L_{j}^{c}$ is good then it is serialized at $w_{j}^{c}$ and the proof follows. If $L_{j}^{c}$ is bad, then let $\ell_{k}^{d}$ be the last node in $B_{H}^{w_{j}^{c}}$. Since $L_{j}^{c}$ is bad it is serialized by $L_{k}^{d}$. If $L_{k}^{d}$ is serialized after $t_{s}$ then $L_{j}^{c}$ is also serialized after $t_{s}$ and we are done. We continue the proof assuming that $L_{k}^{d}$ is serialized before $t_{s}$, that is, $w_{k}^{d} \rightarrow r_{u}[1]$. By this assumption, there are no good writes which are serialized in the interval starting at $r_{u}[1]$ and ending at $r_{u}[j]$. In this case, Lemma 8 implies that $B_{H}^{w_{j}^{c}} /(j+1) \equiv B_{u} /(j+1)$. Consider $\left(\ell_{j}^{c}, \ell_{\ell}^{e}\right)$, the outgoing edge from $\ell_{j}^{c}$ in $\tilde{B}_{u}$. By Lemma 5 there exists an integer $r, r \geq 0$, such that $\left(\ell_{j}^{c}, \ell_{\ell}^{e+r}\right)$ is an edge in $H^{w_{j}^{c}}$. By Lemma $10 L_{j}^{c}$ is serialized after $L_{\ell}^{e+r}$. We now show that if $r>0$, then $L_{\ell}^{e+r}$ is serialized after $t_{s}$, and therefore $L_{j}^{c}$ is also serialized after $t_{s}$. Later we show that indeed $r>0$. If $r>0$ then $r>1$ since a writer never uses the same address twice in a row. Since $\ell_{\ell}^{e}$ is the current label read at $\tilde{r}_{u}[\ell], \tilde{r}_{u}[\ell] \rightarrow w_{\ell}^{e+1}$. Since $w_{\ell}^{e+1} \rightarrow r_{\ell}^{e+r}[1]$ we get that $\tilde{r}_{u}[\ell] \rightarrow r_{\ell}^{e+r}[1]$ which implies that $L_{\ell}^{e+r}$ is serialized after $t_{s}$. Therefore $L_{j}^{c}$ is also serialized after $t_{s}$. The proof is completed by showing that $r>0$. Assume by way of contradiction that $r=0$, that is $\left(\ell_{j}^{c}, \ell_{\ell}^{e}\right)$ is an edge in $H^{w_{j}^{c}}$. Since $L_{j}^{c}$ is bad, $\ell_{\ell}^{e}$ is not last in $B_{H}^{w_{j}^{c}} / j$. Since $B_{u} /(j+1) \equiv B_{H}^{w_{j}^{c}} /(j+1)$ and $B_{u} \subset \tilde{G}_{u}$, the edge excluding $\left(\ell_{j}^{c}, \ell_{\ell}^{e}\right)$ from $B_{H}^{w_{j}^{c}}$ also excludes $\left(\ell_{j}^{c}, \ell_{\ell}^{e}\right)$ from $\tilde{B}_{u}$, contradiction to the definition of $\ell_{j}^{c}$. 
Case 4: $B_{u} \not \tilde{B}_{u}$ and $B_{u} \not \subset \tilde{G}_{u}$.

Let $i$ be the smallest $i d$ such that $\ell_{i}^{a}$ is in $B_{u}, \ell_{i}^{b} \in \tilde{G}_{u}$ and $\ell_{i}^{a} \not \ell_{i}^{b}$. According to the reader protocol, $S_{u}$ returns $\ell_{i}^{b}$. We show that $L_{i}^{b}$ is serialized after $t_{s}$. Since $\ell_{i}^{b} \not \ell_{i}^{a}, w_{i}^{a} \rightarrow r_{u}[i] \rightarrow$ $w_{i}^{b} \rightarrow \tilde{r}_{u}[i]$, in particular $r_{u}[1] \rightarrow w_{i}^{b}$. If $L_{i}^{b}$ is good, then it is serialized after $t_{s}$. Hence for the rest of Case 4 , we assume that $L_{i}^{b}$ is bad. We show that there exists a good write action $L_{j}^{c}$ which is serialized after $t_{s}$, and $L_{i}^{b}$ is either serialized by $L_{j}^{c}$ or it is serialized after $L_{j}^{c}$. Consider the following cases:

Case 4.1: $\ell_{i}^{a} \in B_{H}^{r_{u}[i]}$.

Since $L_{i}^{b}$ is bad, $B_{H}^{w_{i}^{b}} / i \not \equiv B_{i}^{b} / i$, hence Lemma 8 implies that there exists a good write action $L_{j}^{c}$ such that $j<i$ and $r_{i}^{b}[j] \rightarrow w_{j}^{c} \rightarrow w_{i}^{b}$. Since $w_{i}^{a} \rightarrow r_{i}^{b}[j]$ we get that $w_{i}^{a} \rightarrow w_{j}^{c} \rightarrow w_{i}^{b}$.

Now we use $L_{j}^{c}$ to show that $L_{i}^{b}$ is serialized after $t_{s}$. Since $L_{i}^{b}$ is bad, it is serialized by the label that was last in $B_{H}^{w_{i}^{b}}$. Since $w_{j}^{c} \rightarrow w_{i}^{b}$, the last label in $B_{H}^{w_{i}^{b}}$ is either $\ell_{j}^{c}$ or the label of another logical write action which is serialized after $L_{j}^{c}$. The proof is completed by showing that $L_{j}^{c}$ is serialized after $r_{u}[i]$, which implies that $L_{i}^{b}$ is also serialized after $r_{u}[i]$ and therefore after $t_{s}$. Since $L_{j}^{c}$ is good, it is serialized at $w_{j}^{c}$. Therefore it is enough to show that $r_{u}[i] \rightarrow w_{j}^{c}$. Note that since $w_{i}^{a} \rightarrow w_{j}^{c}, \ell_{i}^{a} \in H^{w_{j}^{c}}$. However, since $\ell_{j}^{c}$ is last in $B_{H}^{w_{j}^{c}}$ and $i>j$, Lemma 4(a) implies that $\ell_{i}^{a} \notin B_{H}^{w_{j}^{c}}$. Therefore Lemma 1 implies that for any $t$ after the occurrence time of $w_{j}^{c}, \ell_{i}^{a} \notin B_{H}^{t}$. By the assumption of this case $\ell_{i}^{a} \in B_{H}^{r_{u}[i]}$ therefore $r_{u}[i] \rightarrow w_{j}^{c}$.

Case 4.2: $\ell_{i}^{a} \notin B_{H}^{r_{u}[i]}$.

By the definition of $i$ in Case $4, \ell_{i}^{a} \in B_{u}$. On the other hand, by Case $4.2 \ell_{i}^{a} \notin B_{H}^{r_{u}}[i]$, therefore $B_{H}^{r_{r}[i]} /(i+1) \not \equiv B_{u} /(i+1)$. By Lemma 8 there exists a good write action, $L_{j}^{c}$, $j<i$, such that $r_{u}[j] \rightarrow w_{j}^{c} \rightarrow r_{u}[i] . L_{j}^{c}$ is good, therefore it is serialized at $w_{j}^{c}$. Since $w_{j}^{c} \rightarrow r_{u}[i] \rightarrow w_{i}^{b}$, we get $w_{j}^{c} \rightarrow w_{i}^{b}$, and therefore $L_{i}^{b}$ is serialized either by $L_{j}^{c}$ or later. Since $w_{j}^{c}$ occurs after $t_{s}, L_{i}^{b}$ is serialized after $t_{s}$ too.

\section{Multi-Writer out of Single-Reader Registers}

\subsection{Description}

In this section we present an implementation in which the physical registers are atomic, $(1,1)$-registers. In this implementation readers and writers share the same protocol which is obtained by modifying the writer protocol of the $(1, n)$ implementation. Throughout this section we assume that the $i d s$ of the readers are larger than the $i d s$ of the writers. The reader protocol contains an extra return statement which terminates its execution. Communication is two-sided, as implied by the fact that the writer and reader protocols are the same and as proved necessary by the recent result of [ITV92].

Processor $P_{i}$ communicates with processor $P_{j}$, where $i \neq j$, by writing into a $(1,1)$ atomic register denoted by $R_{i, j}$ from which $P_{j}$ reads. A physical read action executed by $P_{i}$ from $R_{j, i}$ is denoted by $r_{i}[j]$; while $w_{i}[j]$ denotes a physical write action by $P_{i}$ to $R_{i, j}$. Logical action number $a$ of $P_{i}$, where $P_{i}$ is either a writer or a reader, is denoted by $L_{i}^{a}$. Since we use $(1,1)$ registers, some single physical actions of the $(1, n)$ implementation are now replaced by $n$ physical actions (for example $w_{i}^{a}$ is replaced by $\left.w_{i}^{a}[1] \ldots w_{i}^{a}[n]\right)$. For reasons we explain later each register contains five successive labels (and their corresponding values). The five labels are called new, current, previous, old and ancient. 


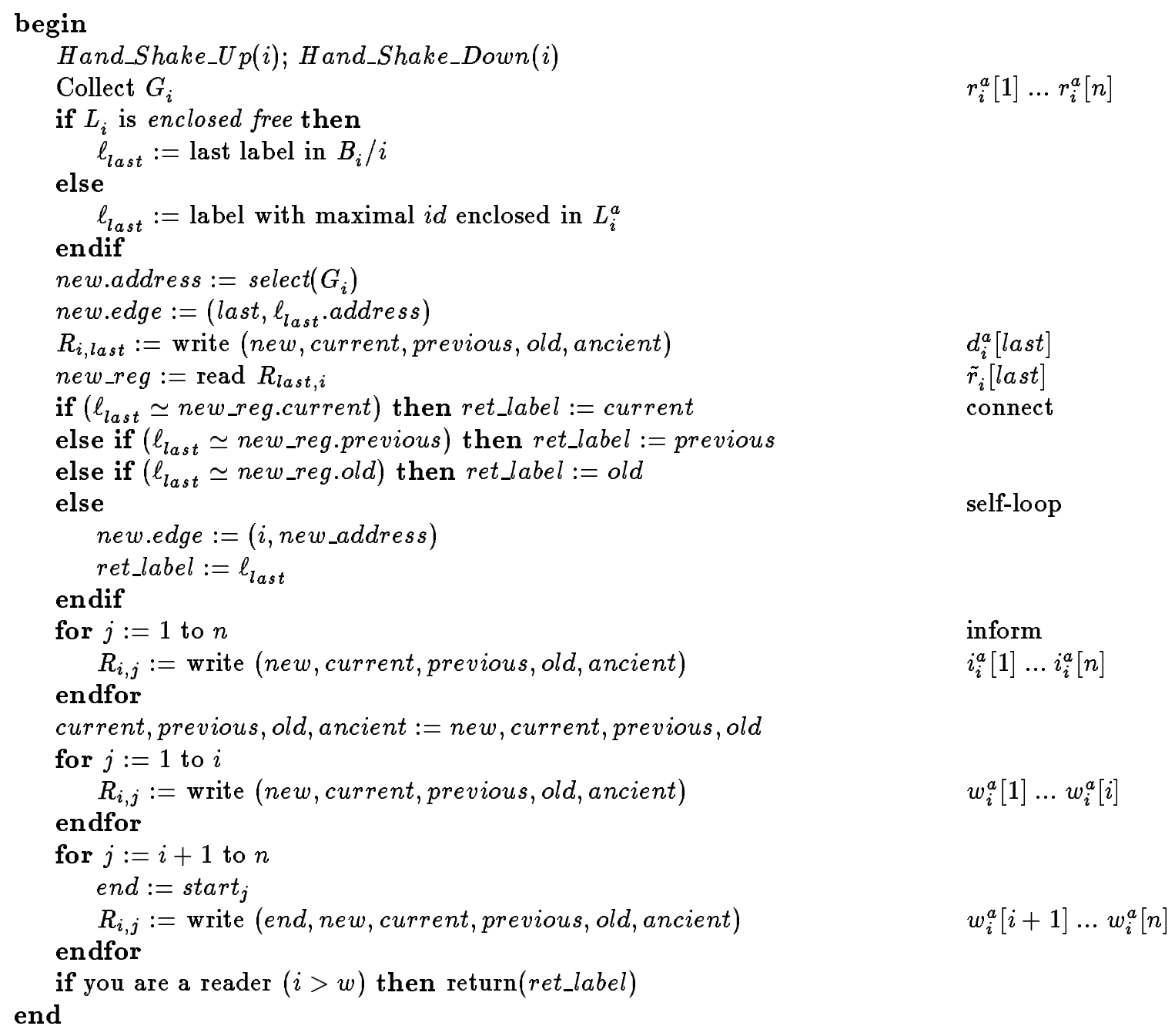

FIguRE 6 . The protocol for $P_{i}$ in the $(1,1)$ implementation 
Procedure Hand_Shake_Up(i)

begin

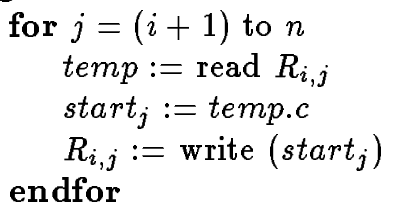

$$
\begin{aligned}
& r_{i}^{a}[i+1] . c, \ldots, r_{i}^{a}[n] . c \\
& w_{i}^{a}[i+1] . s, \ldots, w_{i}^{a}[n] . s
\end{aligned}
$$

end

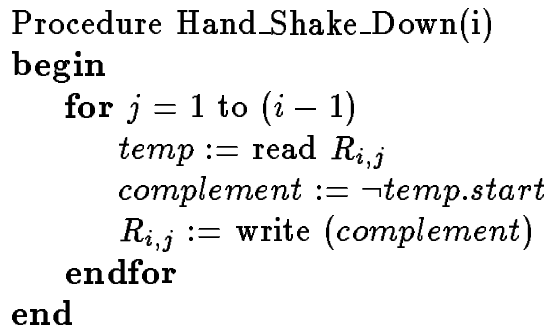

$$
\begin{aligned}
& r_{i}^{a}[1] . s, \ldots, r_{i}^{a}[i-1] . s \\
& w_{i}^{a}[1] . c, \ldots, w_{i}^{a}[i-1] . c
\end{aligned}
$$

FIgURE 7. The Hand_Shake procedures

Immediately after the occurrence of $w_{i}^{a}[j]$ the current label in $R_{i, j}$ holds $\ell_{i}^{a}$, the label computed by $L_{i}^{a}$, while previous, old and ancient hold $\ell_{i}^{a-1}, \ell_{i}^{a-2}$ and $\ell_{i}^{a-3}$ respectively.

The serialization interval of logical action $L_{i}^{a}$ is some interval (to be precisely defined later) enclosed within its execution interval and in which its serialization time lies. Our goal in this protocol is to enable each processor to compute its target label after collection of a single precedence graph. In case there exists no lower indexed processor that executes many actions overlapping the collection phase we show that the frontal branch of the history graph at the beginning of $L_{i}^{a}$ 's serialization interval is a subgraph of $G_{i}^{a}$. In this case the last node of $B_{i}^{a} / i$ is a valid target. If however there exists a lower indexed processor that executes many overlapping actions then the last node of $B_{i}^{a} / i$ may not be used as a target. In this situation the processor identifies one of these overlapping actions of a lower-indexed processor and uses its label as its target. The overlapping actions identified during execution of $L_{i}^{a}$ are called enclosed within $L_{i}^{a}$. The hand-shake mechanism enables $L_{i}^{a}$ to detect enclosed actions. In section 5.2 we formally define the enclosed relation and give a detailed explanation on how the hand-shake procedures work. The code of the protocol appears in Figures 6 and 7: We now describe the code assuming logical action $L_{i}^{a}$ is executed. Action $L_{i}^{a}$ is started with execution of procedures $H_{a n d} S h a k e_{-} U p$ and Hand_Shake_Down. Procedure Hand_Shake_Up enables detection of $L_{i}^{a}$ as enclosed by actions of processors with $i d>i$. The physical actions executed during Hand_Shake_Up are denoted by $r_{i}^{a}[i+1] . c, w_{i}^{a}[i+1] . s \ldots r_{i}^{a}[n] . c, w_{i}^{a}[n] . s$. Procedure Hand_Shake_Down enables $L_{i}^{a}$ to detect enclosed actions of processors with id $<i$. The physical actions executed during Hand_Shake_Down are denoted by $r_{i}^{a}[1] . s, w_{i}^{a}[1] . c \ldots r_{i}^{a}[i-1] . s, w_{i}^{a}[i-1] . c$.

After executing the hand-Shake procedures $L_{i}^{a}$ collects $G_{i}^{a}$ in actions $r_{i}^{a}[1] \ldots r_{i}^{a}[n]$. The graph $G_{i}^{a}$ contains $3 n+1$ nodes - three nodes per processor and the virtual root node. The three labels of $P_{j}$ in $G_{i}^{a}$ are chosen from the five labels read from $R_{j, i}$ in action $r_{i}^{a}[j]$ : If in $G_{i}^{a}$ there exists a label $\ell_{k}$, $j<k<i$, such that $e_{k}$ is directed to the new label of $P_{j}$ in $R_{j, i}$ (i.e. $\ell_{k}$. edge $=\left(j, R_{j, i}\right.$.new.address)) then the three chosen labels are new, current and previous. Otherwise the three labels are current, previous and old. After $G_{i}^{a}$ is collected, $P_{i}$ computes a label called $\ell_{\text {last }}$ towards which the edge of its 
new tentative label is directed. If $L_{i}^{a}$ does not have any enclosed label then $\ell_{\text {last }}$ is the last label in $B_{i}^{a} / i$. Otherwise $\ell_{\text {last }}$ is the enclosed label with the maximal $i d$. Then $P_{i}$ declares its new tentative label to $P_{\text {last }}$ in action $d_{i}^{a}\left[\right.$ last]. In the next step (action $\tilde{r}_{i}^{a}[$ last $]$ ) $P_{i}$ re-reads $R_{\text {last }, i}$. If $\ell_{\text {last }}$ is $\simeq$ to either current, or previous, or old then $L_{i}^{a}$ connects - the tentative new label becomes current. In this case we say that the target label of $\ell_{i}^{a}$ is the label read in $\tilde{r}_{i}^{a}$ [last], which is $\simeq$ to $\ell_{\text {last }}$. Otherwise ( $L_{i}^{a}$ loops), $e_{i}^{a}$ forms a self-loop.

The address of the tentative new label is obtained by the function select that considers all five labels collected from each processor. Since every register contains five labels, the addresses of every consecutive five labels are distinct. Therefore, if $\ell_{i}^{a} \simeq \ell_{i}^{a+r}$ and if $r>0$, then $r>4$. The ancient label is used as follows: Consider an execution $E$ in which the edge outgoing from $\ell_{j}^{b}$ is $\left(\ell_{j}^{b}, \ell_{i}^{a}\right)$. In action $w_{j}^{b+4}[i]\left(\ell_{j}^{b}, \ell_{i}^{a}\right)$ is removed from $R_{j, i}$, but it is still present in registers $R_{j, k}$ for $k>i$. At this point, $P_{i}$ may select the address of $\ell_{i}^{a}$ as an address for some new label $\ell_{i}^{a+r}(r>0)$ where $L_{i}^{a+r}$ starts after $L_{j}^{b}$ terminates. If, after $L_{i}^{a+r}$ is completed, $P_{k}$ starts logical action $L_{k}^{c}$ and reads both $R_{j, k}$ and $R_{i, k}$ while collecting $G_{k}^{c}$, then $P_{k}$ adds the edge $\left(\ell_{j}^{b}, \ell_{i}^{a+r}\right)$ to $G_{k}^{c}$. Since in $E, L_{j}^{b} \Rightarrow L_{i}^{a+r}$, this edge violates the precedence relation. The reason for this problem is the fact that due to the use of $(1,1)$ registers, a label "leaves" the system gradually, first for processors with lower $i d$ s and then to those with higher $i d s$. The ancient label provides a window of time during which the old label leaves the system while reuse of its address is delayed. For this reason the ancient label itself is never chosen as a target for new labels.

Action $L_{i}^{a}$ ends with the concluding write stage in which $\ell_{i}^{a}$ is written to each processor in turn. The physical actions in this stage are $w_{i}^{a}[1] \ldots w_{i}^{a}[n]$. The fact that the concluding write of the previous implementation is now replaced with $n$ physical actions causes the following problem: If processor $P_{j}$ directs an edge towards $\ell_{i}^{a}$ before action $w_{i}^{a}[k], k>j$, takes place and if at that point $P_{k}$ reads both $R_{j, k}$ and $R_{i, k}, P_{k}$ may see $\left(\ell_{j}^{b}, \ell_{i}^{a}\right)$ as "hanging in the air", while in fact both its endpoints join the history graph at an earlier stage, hence the graph collected by $P_{k}$ is not updated. To prevent this problem $P_{i}$ informs all processors that $\ell_{i}^{a}$ is its next label before the concluding write stage. This is done during the inform stage of the protocol, in physical actions $i_{i}^{a}[1] \ldots i_{i}^{a}[n]$, in which $\ell_{i}^{a}$ is written in the new label field, of $R_{i, 1} \ldots R_{i, n}$ respectively, while all other fields in these registers remain unchanged. If in action $L_{k}^{c}, P_{k}$ sees some edge $e_{j}, i<j<k$, directed towards a new label $\ell_{i}^{a}$, $P_{k}$ concludes that $\ell_{i}^{a}$ can safely be added to $G_{k}^{c}$.

During execution of $L_{i}^{a}, P_{i}$ computes a label called ret_label. This label is used only if $P_{i}$ is a reader, in this case the value returned by $L_{i}^{a}$ is the value corresponding to ret_label; this value also becomes the value corresponding to $\ell_{i}^{a}$. This is the only place in the implementation in which a value corresponding to one label is copied to another label. Since there are five labels in each register, and each label contains three fields each of which is of size $\log n+O(1)$ bits, the space complexity of the $(1,1)$ implementation is $O(\log n)$. The time complexity is $5 n+O(1)$.

\subsection{Serialization Scheme}

Serialization in the $(1,1)$ protocol is done once more using a history graph. The history graph contains labels of writers and readers. The time $\ell_{i}^{a}$ joins the history graph, which is called the joining time of $\ell_{i}^{a}$ should satisfy the following requirements:

1. The joining time of $\ell_{i}^{a}$ lies within the interval $\left[w_{i}^{a}[i+1], w_{i}^{a}[n]\right]$.

2. If $\left(\ell_{i}^{a}, \ell_{j}^{b}\right), i>j$, is an edge in $H$ then the joining time of $\ell_{i}^{a}$ is after the joining time of $\ell_{j}^{b}$.

These requirements are fulfilled by the following formal inductive definition: 
Definition 5: Let $\tau$ be the minimal time interval between any two successive physical actions and let $\epsilon=\tau / n$. Let $L_{i}^{a}$ be a logical action where $t_{1}$ is the occurrence time of $w_{i}^{a}[n]$ and $t_{2}$ is the earliest joining time of any label whose target is $\ell_{i}^{a}$. The joining time of $\ell_{i}^{a}$ is defined to be $\min \left(t_{1}, t_{2}-\epsilon\right)$. If $\ell_{i}^{a}$ is the target label of $\ell_{j}^{b}$ and the joining time of $\ell_{i}^{a}$ is determined according to the joining time of $\ell_{j}^{b}$ then we say that $\ell_{i}^{a}$ joins the history graph by $\ell_{j}^{b}$.

Using this definition we now define the history graph and the set of good actions:

Definition 6: Let $E$ be an execution of the system. The History graph $H$ of $E$ is defined as follows:

1. $H^{0}$ is the History graph at time 0 (before the execution begins). It contains only the root node $-\ell_{0}^{0}$.

2. Let $L_{i}^{a}$ be an arbitrary logical action. Let $t$ be the joining time of $\ell_{i}^{a}$. $H^{t}$ is the graph obtained from $H^{t-\epsilon}$ by adding node $\ell_{i}^{a}$, and the directed edge $e_{i}^{a}$ as follows: If $L_{i}^{a}$ connects then $e_{i}^{a}$ is directed towards the target label of $\ell_{i}^{a}$. Otherwise ( $L_{i}^{a}$ loops), $e_{i}^{a}$ is a self-loop.

Definition 7: Action $L_{i}^{a}$ is good if $\ell_{i}^{a}$ is last in $B_{H}^{t}$ where $t$ is the joining time of $\ell_{i}^{a}$.

We now define the enclosed relation; we first give an operational definition and then motivate it by some intuitive explanation: To detect enclosed actions, every physical register $R_{i, k}, i<k$, is augmented with two hand-shake bits called start and end, while every $R_{i, j}, i>j$ is augmented with one hand-shake bit called complement. Action $L_{i}^{a}$ detects an enclosed action $L_{j}^{b}, j<i$, as follows: In actions $r_{j}^{b}[i] . c$ and $w_{j}^{b}[i] . s$ (executed in procedure Hand_Shake_Up during $L_{j}^{b}$ ) the complement bit of $R_{i, j}$ is read and copied to the start bit of $R_{j, i}$. Then in action $w_{j}^{b}[i]$ this value is copied to the end bit of $R_{j, i}$. In contrast, in actions $r_{i}^{a}[j] . s$ and $w_{i}^{a}[j] . c$ (executed in Hand_Shake_Down during $L_{i}^{a}$ ) the start bit of $R_{j, i}$ is read and its complement is written into the complement bit of $R_{i, j}$. If in action $r_{i}^{a}[j]$, executed during the collect stage of $L_{i}^{a}$, it is found that the values of the start and end bits in $R_{j, i}$ are both equal to the complement bit in $R_{i, j}$, then $L_{j}^{b}$ is enclosed within $L_{i}^{a}$.

Definition 8: Let $L_{j}^{b}$ and $L_{i}^{a}, j<i$, be some logical actions. Action $L_{j}^{b}$ and label $\ell_{j}^{b}$ are enclosed within $L_{i}^{a}$ if $\ell_{j}^{b}$ is the current label in $R_{j, i}$ at $r_{i}^{a}[j]$ and the start and the end bits read in $r_{i}^{a}[j]$ are both equal to the complement bit in $R_{i, j}$. Action $L_{i}^{a}$ is enclosed free if it has no enclosed labels.

This operational definition can be intuitively motivated as follows: The protocol is designed so that the serialization time of action $L_{i}^{a}$ lies within the interval starting at $r_{i}^{a}[1] . s$ (the first operation in the Hand_Shake_Down procedure) and ending at the joining time of $\ell_{i}^{a}$. This interval is called the serialization interval of $L_{i}^{a}$. If while $G_{i}^{a}$ is collected label $L_{j}^{b}$ is detected as enclosed within $L_{i}^{a}$, then it holds that $r_{i}^{a}[1] . s \rightarrow r_{j}^{b}[1] . s$. If under these conditions $L_{i}^{a}$ decides to direct $e_{i}^{a}$ towards $L_{j}^{b}$ then the joining time of $\ell_{i}^{a}$ occurs after the joining time of $\ell_{j}^{b}$ and the serialization interval of $L_{j}^{b}$ is enclosed within the serialization interval of $L_{i}^{a}$. This is formally presented in the following proposition:

Proposition 14: If $L_{j}^{b}$ is enclosed within $L_{i}^{a}$ then $r_{i}^{a}[1] . s \rightarrow r_{j}^{b}[1] . s \rightarrow w_{j}^{b}[i] \rightarrow r_{i}^{a}[j]$.

Proof: Since $L_{j}^{b}$ is enclosed within $L_{i}^{a}$ the complement bit in $R_{i, j}$ is equal to the start bit in $R_{j, i}$. Therefore it is clear that $r_{i}^{a}[j] . s \rightarrow w_{j}^{b}[i] . s$. Since $r_{i}^{a}[1] . s \rightarrow r_{i}^{a}[j] . s($ if $j \neq 1)$ and $w_{j}^{b}[i] . s \rightarrow r_{j}^{b}[1] . s$, we get that $r_{i}^{a}[1] . s \rightarrow r_{j}^{b}[1]$.s. Since $\ell_{j}^{b}$ is the current label in $R_{j, i}$ at $r_{i}^{a}[j]$ it holds that $w_{j}^{b}[i] \rightarrow r_{i}^{a}[j]$, the proof follows. 
Now we can explain why each collected graph contains three labels for each processor: Our goal in this protocol is to enable each processor to compute its target label after collection of a single precedence graph. In case the processor detects some enclosed labels it chooses one of them as its target. Otherwise we require that $B_{H}^{r_{i}^{a}[1]} / i$ should be a subgraph of $G_{i}^{a}$. Assume that $\ell_{j}^{b} \in B_{H}^{r_{i}^{a}[1]} / i$. It can be shown that if $P_{j}$ does not generate any enclosed label then it can produce at most two labels in addition to $\ell_{j}^{b}$ before the occurrence time of $r_{i}^{a}[j]$. The first label is produced by an action which starts before the occurrence of $r_{i}^{a}[1]$ while the second is finished after the occurrence of $r_{i}^{a}[j]$. To ensure that either $R_{j, i}$ consists of some enclosed label or that $\ell_{j}^{b} \in G_{i}^{a}$ each collected graph contains three labels of every processor.

We now define the serialization time for the logical actions. Logical write actions are serialized independently of the logical read actions. A good write action is serialized at its joining time. A bad write action $L_{j}^{b}$ is serialized by the last label in $B_{H}^{t} / w+1$ (i.e. the last label of a writer) where $t$ is $L_{j}^{b}$ 's joining time. In Theorem 21 we prove that each logical write action is serialized within its serialization interval.

Definition 9: Let $L_{i}^{a}$ be a logical write action whose joining time is $t$.

1. If $L_{i}^{a}$ is good then $L_{i}^{a}$ is serialized at $t$.

2. If $L_{i}^{a}$ is bad and $\ell_{j}^{b}$ is the last node of $B_{H}^{t} /(w+1)$ then $L_{i}^{a}$ is serialized by $L_{j}^{b}$, that is, before $L_{j}^{b}$ and after any other physical action that precedes the joining time of $L_{j}^{b}$. In case several logical write actions are serialized by the same logical action they are serialized in ascending order of their $i d s$, starting with the lowest $i d$ and ending with the highest.

Read actions are serialized according to the labels they return. The serialization time of a logical read action $L_{i}^{a}$ is defined as follows:

Definition 10: Let $L_{i}^{a}$ be a logical read action whose ret_label is $\ell_{j}^{b}$. If $L_{j}^{b}$ is serialized before $r_{i}^{a}[1] . s$ then $L_{i}^{a}$ is serialized at $r_{i}^{a}[1] . s$. Otherwise, $L_{i}^{a}$ is serialized by $L_{j}^{b}$, that is, after $L_{j}^{b}$ and before any physical action which occurs after $L_{j}^{b}$, and any logical action which is serialized after $L_{j}^{b}$. In case several logical read actions are serialized by the same logical action they are serialized in ascending order of their $i d s$.

Based on this definition it is very easy to show for a logical read action $L_{i}^{a}$ that is serialized within its serialization interval. The fact that $L_{i}^{a}$ is serialized after $r_{i}^{a}[1] . s$ follows immediately from Definition 10 . In the next proposition it is shown that the serialization time of $L_{i}^{a}$ is not later then its joining time and therefore the requirement that $L_{i}^{a}$ is serialized within the interval $\left[r_{i}^{a}[1] . s, t\right]$, where $t$ is the joining time of $\ell_{i}^{a}$ is preserved. The proof is by induction.

Proposition 15: Let $t$ be the joining time of $L_{i}^{a}$. If $\ell_{j}^{b}$ is the ret_label of $L_{i}^{a}$ then $L_{j}^{b}$ is serialized before $t$.

\subsection{Correctness Proof}

We begin this section with several auxiliary lemmas which are used in the proof of Theorem 21 in which it is proved that the serialization definition of the write actions satisfies the requirements.

Proposition 16: The last label in $B_{i}^{a} / i$ is not a new label. 
Proof: By the definition of $B_{i}^{a} / i$, the last label of $B_{i}^{a} / i$ has no incoming edge from a label whose $i d$ is smaller then $i$. Therefore, by the construction of $G_{i}^{a}$, the last label in $B_{i}^{a} / i$ is either current or previous or old.

LEMma 17: If $\left(\ell_{i}^{a}, \ell_{j}^{b}\right), i>j$, is an edge in $G_{k}^{c}$ then there exists some integer $r, r \geq 0$ such that $\left(\ell_{i}^{a}, \ell_{j}^{b+r}\right)$ is an edge in $H$.

Proof: By the protocol, if $\left(\ell_{i}^{a}, \ell_{j}^{b}\right)$ is an edge in $G_{k}^{c}$, where $i>j$, then $L_{i}^{a}$ connects, and its target label is some label of $P_{j}, \ell_{j}^{b+r}$. Obviously $\ell_{j}^{b+r}$.address $=\ell_{j}^{b}$.address. To prove the lemma we show that $r \geq 0$. Assume by way of contradiction that $r<0$. Since the addresses of every five consecutive labels are distinct and $\ell_{j}^{b+r}$.address $=\ell_{j}^{b}$.address, we get that $r<-4$. Label $\ell_{i}^{a}$ is one of the five labels in $R_{i, j}$ throughout the interval $\left[d_{i}^{a}[j], w_{i}^{a+4}[j]\right)$. We prove the lemma by showing that $d_{i}^{a}[j] \rightarrow r_{j}^{b}[i] \rightarrow w_{i}^{a+4}[j]$, contradictory to the definition of the function select (executed during $L_{j}^{b}$ ).

First we show that $d_{i}^{a}[j] \rightarrow r_{j}^{b}[i]$. The old, previous and current labels in $R_{j, i}$ at $w_{j}^{b-2}[i]$ are $\ell_{j}^{b-4}$, $\ell_{j}^{b-3}$ and $\ell_{j}^{b-2}$ respectively. Since $r<-4, \ell_{j}^{b+r}$ is neither old nor previous nor current in $R_{j, i}$ at $w_{j}^{b-2}[i]$. Since $\ell_{j}^{b+r}$ is one of these labels in $R_{j, i}$ at $\tilde{r}_{i}^{a}[j]$ we get that $\tilde{r}_{i}^{a}[j] \rightarrow w_{j}^{b-2}[i]$. By the protocol $d_{i}^{a}[j] \rightarrow \tilde{r}_{i}^{a}[j]$ and $w_{j}^{b-2}[i] \rightarrow r_{j}^{b}[i]$, hence $d_{i}^{a}[j] \rightarrow r_{j}^{b}[i]$.

The fact that $r_{j}^{b}[i] \rightarrow w_{i}^{a+4}[j]$ is proved as follows:

1. $r_{j}^{b}[i] \rightarrow i_{j}^{b}[k]$ (according to the protocol)

2. $i_{j}^{b}[k] \rightarrow r_{k}^{c}[j]\left(\right.$ since $\left.\ell_{j}^{b} \in G_{k}^{c}\right)$

3. $r_{k}^{c}[j] \rightarrow r_{k}^{c}[i]$ (since $i>j$ and $G_{k}^{c}$ is collected in ascending order)

4. $r_{k}^{c}[i] \rightarrow w_{i}^{a+3}[k]\left(\right.$ since $\left.\ell_{i}^{a} \in G_{k}^{c}\right)$

5. $w_{i}^{a+3}[k] \rightarrow w_{i}^{a+4}[j]$ ( $P_{i}$ works in sequential manner)

Since the right hand side of each relation is the left hand side of the next one we get $r_{j}^{b}[i] \rightarrow w_{i}^{a+4}[j]$. In conclusion: $d_{i}^{a}[j] \rightarrow r_{j}^{b}[i] \rightarrow w_{i}^{a+4}[j]$, a contradiction.

LEMma 18: Let $L_{k}^{c}$ be an enclosed free action and let $t$ be the joining time of $\ell_{k}^{c}$. Every node of $B_{k}^{c} / k$ belongs to $H^{t}$.

Proof: We first show that the last label of $B_{k}^{c} / k$ satisfies the lemma: Let $\ell_{i}^{a}$ be the last label of $B_{k}^{c} / k$. By Proposition $16, \ell_{i}^{a}$ is not the new label in $R_{i, k}$ at $r_{k}^{c}[i]$. Therefore $\ell_{i}^{a}$ is either current or previous or old. If $w_{i}^{a}[n]$ occurs before $t$, as in the case when $\ell_{i}^{a}$ is either previous or old, we are done. The only remaining case is when $w_{i}^{a}[n]$ occurs after $t$. In this case $\ell_{i}^{a}$ is current in $R_{i, k}$ at $r_{k}^{c}[i]$. Since $L_{k}^{c}$ is enclosed free the label $\ell_{\text {last }}$ computed during $L_{k}^{c}$ is the last label in $B_{k}^{c} / k$, namely $\ell_{i}^{a}$. We now show that $w_{i}^{a}[k] \rightarrow \tilde{r}_{k}^{c}[i] \rightarrow w_{i}^{a}[n]$, which implies that $\ell_{i}^{a}$ is the target label of $\ell_{k}^{c}$, that hence its joining time is no later then the joining time of $\ell_{i}^{a}$, namely $t$. The first relation is proved as follows: Since $\ell_{i}^{a}$ is current in $R_{i, k}$ at $r_{k}^{c}[i]$, we get that $w_{i}^{a}[k] \rightarrow r_{k}^{c}[i]$. According to the protocol $r_{k}^{c}[i] \rightarrow \tilde{r}_{k}^{c}[i]$ thus $w_{i}^{a}[k] \rightarrow \tilde{r}_{k}^{c}[i]$. To prove that $\tilde{r}_{k}^{c}[i] \rightarrow w_{i}^{a}[n]$ note that by Definition 5 , the joining time of $\ell_{k}^{c}$ is within the interval $\left[w_{k}^{c}[k], w_{k}^{c}[n]\right]$. According to the protocol $\tilde{r}_{k}^{c}[i] \rightarrow w_{k}^{c}[k]$, hence $\tilde{r}_{k}^{c}[i]$ is before $t$. Since we assume that $w_{i}^{a}[n]$ occurs after $t$, we get that $\tilde{r}_{k}^{c}[i] \rightarrow w_{i}^{a}[n]$. 
To complete the proof it suffices to show that if $\left(\ell_{i}^{a}, \ell_{j}^{b}\right)$ is an edge in $B_{k}^{c} / k$, and if the joining time of $\ell_{i}^{a}$ is before $t$, then the joining time of $\ell_{j}^{b}$ is also before $t$. By Definition 5 the joining time of $\ell_{i}^{a}$ is after the occurrence time of $w_{i}^{a}[i]$. Since according to the protocol $\tilde{r}_{i}^{a}[j] \rightarrow w_{i}^{a}[i]$ and since the joining time of $\ell_{i}^{a}$ is before $t$, we get that $\tilde{r}_{i}^{a}[j]$ occurs before $t$. By Lemma 17 there exists some $r, r \geq 0$, such that $\ell_{j}^{b+r}$ is the target label of $\ell_{i}^{a}$. If $r=0$ then $\ell_{j}^{b}$ is the target label of $\ell_{i}^{a}$ which implies by Definition 5 that the joining time of $\ell_{j}^{b}$ is before $t$, and we are done. If $r>0$ then $w_{j}^{b}[n] \rightarrow w_{j}^{b+r}[i] \rightarrow \tilde{r}_{i}^{a}[j]$. In this case $w_{j}^{b}[n]$ occurs before $t$ and again by Definition 5 the joining time of $\ell_{j}^{b}$ is before $t$.

LEMma 19: If $\ell_{j}^{b}$ is the label with the maximal $i d$ enclosed in $L_{i}^{a}$, then the joining time of $\ell_{j}^{b}$ is before the joining time of $\ell_{i}^{a}$.

Proof: By the protocol $\ell_{j}^{b}$ is the label $\ell_{\text {last }}$ computed during $L_{i}^{a}$. By Definition 5 the joining time of $\ell_{j}^{b}$ occurs within the interval $\left[w_{j}^{b}[j], w_{j}^{b}[n]\right]$. To prove the lemma we show that either $w_{j}^{b}[n] \rightarrow w_{i}^{a}[1]$ or $\ell_{j}^{b}$ is the target label of $\ell_{i}^{a}$ and hence, by Definition 5 , the joining time of $\ell_{j}^{b}$ is before the joining time of $\ell_{i}^{a}$. If $w_{j}^{b}[n] \rightarrow \tilde{r}_{i}^{a}[j]$ then $w_{j}^{b}[n] \rightarrow w_{i}^{a}[1]$ and we are done. Assume $\tilde{r}_{i}^{a}[j] \rightarrow w_{j}^{b}[n]$. Since $\ell_{j}^{b}$ is enclosed in $L_{i}^{a}$, Definition 8 implies that $w_{j}^{b}[i] \rightarrow r_{i}^{a}[j]$. Since $r_{i}^{a}[j] \rightarrow \tilde{r}_{i}^{a}[j]$ we get $w_{j}^{b}[i] \rightarrow \tilde{r}_{i}^{a}[j] \rightarrow w_{j}^{b}[n]$. Therefore $\ell_{j}^{b}$ is the current label in $R_{j, i}$ at $\tilde{r}_{i}^{a}[j]$, that is, $\ell_{j}^{b}$ is the target label of $\ell_{i}^{a}$.

Lemma 20: Let $t$ be the joining time of $\ell_{i}^{a}$.

1. If $B_{H}^{r_{i}^{a}[1] . s} / i \equiv B_{H}^{t} / i$ then

(1.a) $L_{i}^{a}$ is enclosed free.

(1.b) $B_{H}^{r_{i}^{a}[1] . s} / i$ is a subgraph of $G_{i}^{a}$.

(1.c) $L_{i}^{a}$ is good.

2. For any $t^{\prime} \geq t$, if $\ell_{i}^{a} \in B_{H}^{t^{\prime}}$ then $\ell_{i}^{a+1} \notin H^{t^{\prime}}$.

Proof: By induction on $i$ the $i d$ of the labels.

Base $i=1$ :

(1.a) Every logical action executed by $P_{1}$ is enclosed free. (1.b) For any $t, B_{H}^{t} / 1$ contains a single node, namely $\ell_{0}^{0}$. (1.c) Every action of $P_{1}$ is good.

(2) Every label of $P_{1}$ excludes its previous label from the frontal branch of the history graph.

Step: Assume correctness for $j<i$. We now prove correctness for $i$.

Proof of (1.a): Assume by way of contradiction that $L_{i}^{a}$ is not enclosed free. Let $L_{j}^{b}, j<i$, be the action with maximal $i d$, enclosed in $L_{i}^{a}$. By Proposition 14 it holds that $r_{i}^{a}[1] . s \rightarrow$ $r_{j}^{b}[1] . s$. Since $\ell_{j}^{b}$ is the label with the maximal $i d$ enclosed in $L_{i}^{a}$, Lemma 19 implies that the joining time of $\ell_{j}^{b}, t^{\prime}$, is before $t$. Hence the interval $\left[r_{j}^{b}[1] . s, t^{\prime}\right]$ is enclosed within the interval $\left[r_{i}^{a}[1] . s, t\right]$. Since $B_{H}^{r_{i}^{a}[1] . s} / i \equiv B_{H}^{t} / i$ we get that $B_{H}^{r_{j}^{b}[1] . s} / i \equiv B_{H}^{t^{\prime}} / i$. By the induction assumption, $L_{j}^{b}$ is good. Hence the frontal branch of the history graph is modified at $t^{\prime}$, the joining time of $\ell_{j}^{b}$. Since $j<i, B_{H}^{r_{j}^{b}[1] . s} / i \not \equiv B_{H}^{t^{\prime}} / i$, contradiction. 
Proof of (1.b): In order to show that $B_{H}^{r_{i}^{a}[1] \cdot s} / i$ is a subgraph of $G_{i}^{a}$, we show that every label of $B_{H}^{r_{i}^{a}[1] . s} / i$ belongs to $G_{i}^{a}$ : First we show that every such label is read during collection of $G_{i}^{a}$ either as new or as current or as previous. Let $\ell_{j}^{b}$ be some label in $B_{H}^{r_{i}^{a}[1] . s} / i$. Clearly $i_{j}^{b}[i] \rightarrow r_{i}^{a}[1] . s$ and therefore $i_{j}^{b}[i] \rightarrow r_{i}^{a}[j]$. Since $B_{H}^{r_{i}^{a}[1] . s} / i \equiv B_{H}^{t} / i$ we get that $\ell_{j}^{b} \in B_{H}^{t} / i$ which implies, by induction hypothesis $(2), \ell_{j}^{b+1} \notin H^{t}$. Therefore $w_{j}^{b+1}[n]$ occurs after $t$ which implies that $i_{j}^{b}[i] \rightarrow r_{i}^{a}[j] \rightarrow w_{j}^{b+1}[n]$. Thus $\ell_{j}^{b}$ is either new or current or previous in $R_{j, i}$ at $r_{i}^{a}[j]$. Labels which are either current or previous belong to $G_{i}^{a}$ independently of the rest of the labels in $G_{i}^{a}$. A new label belongs to $G_{i}^{a}$ only if it has an edge incoming from another legal in $G_{i}^{a}$ whose $i d$ is smaller then $i$. If the last label of $B_{H}^{r_{i}^{a}[1] . s} / i$ is in $G_{i}^{a}$ then all the labels in $B_{H}^{r_{i}^{a}[1] . s} / i$ which were read as new have such an incoming edge. Thus, to show that all the labels of $B_{H}^{r_{i}^{a}[1] . s} / i$ are in $G_{i}^{a}$, it suffices to show that the last label of $B_{H}^{r_{i}^{a}[1] . s} / i$ is in $G_{i}^{a}$.

Let $\ell_{j}^{b}$ be the last label in $B_{H}^{r_{i}^{a}[1] . s} / i$. We now show that $w_{j}^{b}[i] \rightarrow r_{i}^{a}[j]$ which implies that $\ell_{j}^{b}$ is either current or previous in $R_{j, i}$ at $r_{i}^{a}[j]$, hence $\ell_{j}^{b} \in G_{i}^{a}$. If the joining time of $\ell_{j}^{b}$ is at $w_{j}^{b}[n]$, then $w_{j}^{b}[n] \rightarrow r_{i}^{a}[1] . s$. Since, by the protocol, $r_{i}^{a}[1] . s \rightarrow r_{i}^{a}[j]$, it holds that $w_{j}^{b}[i] \rightarrow r_{i}^{a}[j]$. Otherwise, $\ell_{j}^{b}$ joins the history graph by another label $\ell_{k}^{c}, j<i \leq k$, whose target label is $\ell_{j}^{b}$. Since $\ell_{j}^{b} \in B_{H}^{r_{i}^{a}[1] . s}$ and $\ell_{j}^{b}$ joins the history graph by $\ell_{k}^{c}$ we get that $\ell_{k}^{c} \in H^{r_{i}^{a}[1] . s}$. The proof is completed by the following relations:

1.a. $w_{j}^{b}[i]=w_{j}^{b}[k]-$ if $k=i$.

1.b. $w_{j}^{b}[i] \rightarrow w_{j}^{b}[k]$ - according to the protocol, assuming $i<k$.

2. $w_{j}^{b}[k] \rightarrow \tilde{r}_{k}^{c}[j]$ - since $\ell_{j}^{b}$ is the target label of $\ell_{k}^{c}$.

3. $\tilde{r}_{k}^{c}[j] \rightarrow r_{i}^{a}[1] . s-$ since $\ell_{k}^{c} \in H^{r_{i}^{a}[1] . s}$.

4. $r_{i}^{a}[1] . s \rightarrow r_{i}^{a}[j]$ - according to the protocol.

which imply $w_{j}^{b}[i] \rightarrow r_{i}^{a}[j]$. Therefore $\ell_{j}^{b} \in G_{i}^{a}$, and $B_{H}^{r_{i}^{a}[1] . s} / i$ is a subgraph of $G_{i}^{a}$.

Proof of (1.c): Assume by way of contradiction that $L_{i}^{a}$ is bad. Since $B_{H}^{r_{i}^{a}[1] . s} / i \equiv B_{H}^{t} / i$, induction hypothesis (1.a) and (1.b) for $i$ imply that $L_{i}^{a}$ is enclosed free and that $B_{H}^{t} / i$ is a subgraph of $G_{i}^{a}$. Since $L_{i}^{a}$ is enclosed free and since $L_{i}^{a}$ is bad, we get that $B_{i}^{a} / i \not \equiv B_{H}^{t} / i$. Let $\ell_{j}^{b}, j<i$, be the label with the maximal $i d$ in their common prefix. Label $\ell_{j}^{b}$ is not last in $B_{i}^{a} / i$ since $B_{H}^{t} / i$ is a subgraph of $G_{i}^{a}$ and $B_{i}^{a} / i \not \equiv B_{H}^{t} / i$. Let $\left(\ell_{k}^{c}, \ell_{j}^{b}\right), k<i$, be the incoming edge to $\ell_{j}^{b}$ in $B_{i}^{a} / i$. We now show that $\left(\ell_{k}^{c}, \ell_{j}^{b}\right)$ is an edge in $H^{t}$ : Since $L_{i}^{a}$ is enclosed free, Lemma 18 implies that $\ell_{k}^{c} \in H^{t}$. By Lemma 17 there exists some $r \geq 0$, such that $\left(\ell_{k}^{c}, \ell_{j}^{b+r}\right)$ is an edge in $H^{t}$. Since $\ell_{j}^{b} \in B_{H}^{t}$, induction hypothesis (2) implies that $\ell_{j}^{b+1} \notin H^{t}$, that is $r=0$. Therefore $\left(\ell_{k}^{c}, \ell_{j}^{b}\right)$ is an edge in $H^{t}$. By the maximality of $\ell_{j}^{b}$, $\left(\ell_{k}^{c}, \ell_{j}^{b}\right) \notin B_{H}^{t} / i$. Let $\left(\ell_{\ell}^{d}, \ell_{j}^{b}\right)$ be the edge excluding $\left(\ell_{k}^{c}, \ell_{j}^{b}\right)$ from $B_{H}^{t} / i$. Since $B_{H}^{t} / i$ is a subgraph of $G_{i}^{a},\left(\ell_{\ell}^{d}, \ell_{j}^{b}\right) \in G_{i}^{a}$, and it excludes $\left(\ell_{k}^{c}, \ell_{j}^{b}\right)$ from $B_{i}^{a} / i$, contradiction.

Proof of (2): Assume by way of contradiction that $\ell_{i}^{a}$ does not satisfy the lemma, that is $\ell_{i}^{a} \in B_{H}^{t_{0}}$ where $t_{0}$ is the joining time of $\ell_{i}^{a+1}$. Since $\ell_{i}^{a} \in B_{H}^{t_{0}}$ it is clear that $L_{i}^{a+1}$ is bad. We reach the required contradiction by showing that $L_{i}^{a+1}$ is good. Since the joining time of $\ell_{i}^{a}$ is before $r_{i}^{a+1}[1] . s$ and $\ell_{i}^{a} \in B_{H}^{t_{0}}$, there are no good actions with $i d$ smaller then $i$ in the interval $\left(r_{i}^{a+1}[1] . s, t_{0}\right)$. Hence $B_{H}^{r_{i}^{a+1}[1] . s} / i \equiv B_{H}^{t_{0}} / i$ which implies, by (1.c), that $L_{i}^{a+1}$ is good. 
The next theorem shows that the protocol satisfies the first design requirement namely that every logical write action is serialized within its serialization interval.

Theorem 21: Every logical write action $L_{i}^{a}$ is serialized within the interval $\left[r_{i}^{a}[1] . s, w_{i}^{a}[n]\right]$.

Proof: By Definition 9, every logical write action is serialized no later then its joining time. Therefore we only have to show that $L_{i}^{a}$ is serialized after $r_{i}^{a}[1]$.s. Let $t$ be the joining time of $\ell_{i}^{a}$. If $L_{i}^{a}$ is good then it is serialized at $t$ and the theorem follows. Assume $L_{i}^{a}$ is bad. By Lemma 20.(1.c) $B_{H}^{r_{i}^{a}[1] . s} / i \not \equiv B_{H}^{t} / i$, hence there exists a good logical write action $L_{j}^{b}$, whose joining time is after $r_{i}^{a}[1] . s$ and before $t$. Therefore $L_{i}^{a}$ is serialized either by $L_{j}^{b}$ or by another logical write action which is serialized after $L_{j}^{b}$ and before $t$, and the theorem follows.

The next lemma shows that the protocol satisfies the second design requirement:

LEMMA 22: If $L_{i}^{a}$ is enclosed free then $B_{H}^{r_{i}^{a}[1]} / i$ is a subgraph of $G_{i}^{a}$.

Proof: To prove this lemma we show that every label in $B_{H}^{r_{i}^{a}[1]} / i$ belongs to $G_{i}^{a}$. Assume by way of contradiction that there are labels in $B_{H}^{r_{i}^{a}[1]} / i$ that do not belong to $G_{i}^{a}$, and let $\ell_{j}^{b}, j<i$, be the label with maximal $i d$ among them. Let $\ell_{j}^{b+r}, \ell_{j}^{b+r-1}$ and $\ell_{j}^{b+r-2}$ be the three labels of $P_{j}$ in $G_{i}^{a}$. By the contradiction assumption $\ell_{j}^{b} \notin G_{i}^{a}$, therefore either $r<0$ or $r \geq 3$. We reach the required contradiction by the following case analysis:

Case 1: $r<0$.

By Definition 5 the joining time of $\ell_{j}^{b}$ is after $i_{j}^{b}[i]$. Since $\ell_{j}^{b} \in B_{H}^{r_{i}^{a}[1]} / i$, we get that $i_{j}^{b}[i] \rightarrow r_{i}^{a}[1]$. According to the protocol $r_{i}^{a}[1] \rightarrow r_{i}^{a}[j]$, hence $i_{j}^{b}[i] \rightarrow r_{i}^{a}[j]$. Since we assume that $r<0$, $r_{i}^{a}[j] \rightarrow w_{j}^{b}[i]$. Therefore $i_{j}^{b}[i] \rightarrow r_{i}^{a}[j] \rightarrow w_{j}^{b}[i]$, and hence $\ell_{j}^{b}$ is the new label in $R_{j, i}$ at $r_{i}^{a}[j]$. Since $r_{i}^{a}[j] \rightarrow w_{j}^{b}[i]$ there is no incoming edge to $\ell_{j}^{b}$ in $H^{r_{i}^{a}[1]}$ from any label whose $i d>i$. Consider the following cases:

Case 1.a: $\ell_{j}^{b}$ is last in $B_{H}^{r_{i}^{a}[1]} / i$

Since there is no incoming edge to $\ell_{j}^{b}$ in $H^{r_{i}^{a}[1]}$ from any label whose $i d>i$ and since $\ell_{j}^{b}$ is last in $B_{H}^{r_{i}^{a}[1]} / i$, we get that $\ell_{j}^{b}$ is last in $B_{H}^{r_{i}^{a}[1]}$. Therefore $\ell_{j}^{b}$ joins the history graph at $w_{j}^{b}[n]$, that is $w_{j}^{b}[n] \rightarrow r_{i}^{a}[1]$, contradiction.

Case 1.b: $\ell_{j}^{b}$ is not last in $B_{H}^{r_{i}^{a}[1]} / i$.

Let $\left(\ell_{k}^{c}, \ell_{j}^{b}\right), i>k>j$, be the incoming edge to $\ell_{j}^{b}$ in $B_{H}^{r_{i}^{a}[1]} / i$. Since $k>j$ and $\ell_{j}^{b}$ is the label with the maximal $i d$ that does not satisfy the lemma, $\ell_{k}^{c} \in G_{i}^{a}$. Since $\ell_{j}^{b}$ is the new label in $R_{j, i}$ at $r_{i}^{a}[j]$, by the construction of $G_{i}^{a}, \ell_{j}^{b}$ joins $G_{i}^{a}$ by $\ell_{k}^{c}$, that is $r \geq 0$, contradiction.

Case 2: $r \geq 3$

In this case we reach a contradiction by showing that the current label read in action $r_{i}^{a}[j]$, denoted by $\ell_{j}^{c}$, is enclosed within $L_{i}^{a}$. Note that $\ell_{j}^{c}$ is either $\ell_{j}^{b+r}$ or $\ell_{j}^{b+r-1}$. Since $\ell_{j}^{c}$ is the current label read in action $r_{i}^{a}[j]$, we only have to show that $w_{i}^{a} j . c \rightarrow r_{j}^{c}[i] . c$ Since $\ell_{j}^{b} \in B_{H}^{r_{i}^{a}[1]} / i$, Lemma 20(2) implies that $\ell_{j}^{b+1} \notin H_{i}^{r_{i}^{a}[1]}$. Therefore $r_{i}^{a}[1] \rightarrow w_{j}^{b+1}[n]$. Since $w_{i}^{a} j . c \rightarrow r_{i}^{a}[1]$ and since $w_{j}^{b+1}[n] \rightarrow r_{j}^{b+2}[i] . c \quad$ we get that $w_{i}^{a} j . c \rightarrow r_{j}^{b+2}[i]$.c. Since $c \geq b+2$ we get that $w_{i}^{a} j . c \rightarrow r_{j}^{c}[i] . c$. The proof follows. 
The next theorem shows that all graphs collected by the processors are precedence graphs:

Theorem 23: If $\left(\ell_{i}^{a}, \ell_{j}^{b}\right), i>j$, is an edge in $G_{k}^{c}$ then $L_{j}^{b} \Rightarrow L_{i}^{a}$.

Proof: By Lemma $17\left(\ell_{i}^{a}, \ell_{j}^{b+r}\right), r \geq 0$, is an edge in $H$. By Definition 5 the joining time of $\ell_{j}^{b+r}$ is before the joining time of $\ell_{i}^{a}$. We show that $L_{j}^{b} \Rightarrow L_{j}^{b+r}$. Since $L_{j}^{b} \Rightarrow L_{i}^{a}$ the proof follows. Consider the following cases:

Case 1: $i \leq w$

In this case $\ell_{j}^{b+r}$ and $L_{i}^{a}$ are both write actions. If $L_{i}^{a}$ is good or $L_{i}^{a}$ and $L_{j}^{b+r}$ are both bad Definition 9 implies that $L_{i}^{a}$ is serialized after $L_{j}^{b+r}$. If $L_{i}^{a}$ is bad while $L_{j}^{b+r}$ is good then since $\left(\ell_{i}^{a}, \ell_{j}^{b+r}\right)$ is an edge in $H$, there at least one good write action which joins the history graph after $L_{j}^{b+r} . L_{i}^{a}$ is serialized by one of this actions, therefore it is serialized after $L_{j}^{b+r}$.

Case 2: $j \leq w<i$

In this case the value written by $L_{j}^{b+r}$ is returned by $L_{i}^{a}$. By Definition $10, L_{j}^{b+r} \Rightarrow L_{i}^{a}$.

Case 3: $j>w$

By Definition $10 L_{j}^{b+r} \Rightarrow L_{i}^{a}$.

The next lemma is used in the correctness proof of the reader protocol.

LEMma 24: Let $L_{i}^{a}, i>w$, be an enclosed free read action. If $\ell_{j}^{b}, j \leq w$, is the last label in $B_{i}^{a} / i$ and $\ell_{j}^{b} \notin B_{H}^{r_{i}^{a}[1]} / i$ then $L_{j}^{b}$ is serialized after $r_{i}^{a}[1]$.

Proof: $\quad$ Since $\ell_{j}^{b} \in B_{i}^{a} /(w+1)$ but $\ell_{j}^{b} \notin B_{H}^{r_{i}^{a}[1]} /(w+1), B_{i}^{a} /(w+1) \not \equiv B_{H}^{r_{i}^{a}[1]} /(w+1)$. Let $\ell_{k}^{c}$ be the label with the maximal $i d$ in the common prefix. By Lemma $22 B_{H}^{r_{i}^{a}[1]} / i$ is a subgraph of $G_{i}^{a}$, hence $\ell_{k}^{c}$ is not last in $B_{i}^{a} /(w+1)$ (otherwise $B_{i}^{a} /(w+1) \equiv B_{H}^{r_{i}^{a}[1]} /(w+1)$ ). Let $\left(\ell_{\ell}^{d}, \ell_{k}^{c}\right)$ be the edge incoming to $\ell_{k}^{c}$ in $B_{i}^{a} /(w+1)$. Let $t$ be the joining time of $\ell_{\ell}^{d}$, we now show that $t$ is after $r_{i}^{a}[1]$. Assume by way of contradiction that $t$ is before $r_{i}^{a}[1]$, that is, $\ell_{\ell}^{d} \in H_{i}^{r_{i}^{a}[1]}$. By Lemma 17, there exists some $r$, $r \geq 0$, such that $\left(\ell_{\ell}^{d}, \ell_{k}^{c+r}\right)$ is an edge in $H^{r_{i}^{a}[1]}$. Since $\ell_{k}^{c} \in B_{H}^{r_{i}^{a}[1]} /(w+1)$, Lemma 20(2) implies that $\ell_{k}^{c \mp 1} \notin H^{r_{i}^{a}[1]} /(w+1)$. Hence $r=0$ and $\left(\ell_{\ell}^{d}, \ell_{k}^{c}\right)$ is an edge in $H^{r_{i}^{a}[1]}$. Let $\left(\ell_{m}^{e}, \ell_{k}^{c}\right), m<\ell$, be the edge in $B_{H}^{r_{i}^{a}[1]}$ excluding $\left(\ell_{\ell}^{d}, \ell_{k}^{c}\right)$ from $B_{H}^{r_{i}^{a}[1]}$. Since $B_{H}^{r_{i}^{a}[1]} / i$ is a subgraph of $G_{i}^{a},\left(\ell_{m}^{e}, \ell_{k}^{c}\right)$ is an edge in $G_{i}^{a}$. Therefore $\left(\ell_{m}^{e}, \ell_{k}^{c}\right)$ excludes $\left(\ell_{\ell}^{d}, \ell_{k}^{c}\right)$ from $B_{i}^{a}$, contradiction. Hence the joining time and (as we show below) the serialization time of $\ell_{\ell}^{d}$ is after $r_{i}^{a}[1]$. If $L_{\ell}^{d} \not \equiv L_{j}^{b}$ then there is a path from from $\ell_{j}^{b}$ to $\ell_{\ell}^{d}$ in $G_{i}^{a}$ (because $\ell_{j}^{b}$ and $\ell_{\ell}^{d}$ are both in $B_{i}^{a} / i$ and $\ell_{j}^{b}$ is last in $B_{i}^{a} / i$ ). By Theorem $23, L_{\ell}^{d} \Rightarrow L_{j}^{b}$, therefore $L_{j}^{b}$ is serialized after $r_{i}^{a}[1]$.

Now we show that $L_{\ell}^{d}$ is serialized after $r_{i}^{a}[1]$. If $B_{H}^{r_{i}^{a}[1]} / \ell \not \equiv B_{H}^{t} / \ell$, then $L_{\ell}^{d}$ is serialized by the good write action that is serialized last before $t$, that is after $r_{i}^{a}[1]$. Assume that $B_{H}^{r_{i}^{a}[1]} / \ell \equiv B_{H}^{t} / \ell$. The following three facts:

1. $B_{H}^{r_{i}^{a}[1]} / i$ is a subgraph of $G_{i}^{a}$, 
2. $\ell_{k}^{c} \in B_{H}^{r_{i}^{a}[1]} / i$

3. $\left(\ell_{\ell}^{d}, \ell_{k}^{c}\right)$ is an edge in $B_{i}^{a} / i$

imply that $\ell_{k}^{c}$ is the last label in $B_{H}^{r_{i}^{a}[1]} / \ell$ (Otherwise the edge incoming to $\ell_{k}^{c}$ in $B_{H}^{r_{i}^{a}[1]} / \ell$ excludes $\left(\ell_{\ell}^{d}, \ell_{k}^{c}\right)$ from $\left.B_{i}^{a} / i\right)$. Since $B_{H}^{r_{i}^{a}[1]} / \ell \equiv B_{H}^{t} / \ell$ it holds that $\ell_{k}^{c}$ is last in $B_{H}^{t} / \ell$ and therefore $L_{\ell}^{d}$ is good. Hence $L_{\ell}^{d}$ is serialized at $t$, that is after $r_{i}^{a}[1]$. The lemma follows.

Theorem 25: Let $L_{i}^{a}$ be a logical read action, and let $L_{j}^{b}$ be the logical write action which wrote the value returned by $L_{i}^{a}$. $L_{j}^{b}$ is the most recent write action serialized before $L_{i}^{a}$.

Proof: We prove this theorem by induction on $i$, the $i d$ of logical readers.

Base: $i=w+1$

Let $\ell_{j}^{b-r}, r \geq 0$, be $\ell_{\text {last }}$ computed in $L_{i}^{a}$. Consider the following cases:

Case 1: $L_{i}^{a}$ is enclosed free

In this case $\ell_{j}^{b-r}$ is the last label in $B_{i}^{a} / i$. By Lemma $22, B_{H}^{r_{i}^{a}[1]} / i$ is a subgraph of $G_{i}^{a}$. We show that either $L_{j}^{b-r}$ is serialized after $r_{i}^{a}[1]$ or $L_{j}^{b-r}$ is the most recent write action serialized before $r_{i}^{a}[1]$. If $\ell_{j}^{b-r} \notin B_{H}^{r_{i}^{a}[1]} / i$ then by Lemma $24 L_{j}^{b-r}$ is serialized after $r_{i}^{a}[1]$. If $\ell_{j}^{b-r} \in B_{H}^{r_{i}^{a}[1]} / i$ then since $B_{H}^{r_{i}^{a}[1]} / i$ is a subgraph of $G_{i}^{a}$, and since $\ell_{j}^{b-r}$ is the last label in $B_{i}^{a} / i, \ell_{j}^{b-r}$ is last in $B_{H}^{r_{i}^{a}[1]} / i$. Hence $L_{j}^{b-r}$ is the most recent write action serialized before $r_{i}^{a}[1]$. Since $L_{j}^{b-r} \Rightarrow L_{j}^{b}$ (if $r>0$ ), the proof follows.

Case 2: $L_{i}^{a}$ is not enclosed free

In this case, $\ell_{j}^{b-r}$ is the label with the maximal $i d$ enclosed in $L_{i}^{a}$. By Proposition 14, it holds that $r_{i}^{a}[1] . s \rightarrow r_{j}^{b-r}[1] . s$. By Theorem $21, L_{j}^{b-r}$ is serialized after $r_{j}^{b-r}[1] . s$. Hence $L_{j}^{b-r}$ is serialized after $r_{i}^{a}[1] . s$. Since $L_{j}^{b-r} \Rightarrow L_{j}^{b}$ (if $r>0$ ) $L_{j}^{b}$ is serialized after $r_{i}^{a}[1] . s$. By Definition $10, L_{i}^{a}$ is serialized by $L_{j}^{b}$, that is, $L_{j}^{b}$ is the most recent write action serialized before $L_{i}^{a}$.

Step: Assume correctness for $m, w<m<i$. We now prove for $i$

If $\ell_{j}^{b}$ is the ret_label of $L_{i}^{a}$ then the proof is identical to the proof of the induction base. Otherwise let $\ell_{k}^{c}, w<k<i$, be the ret_label of $L_{i}^{a}$, and let $\ell_{k}^{c-r}, r \geq 0$, be the label $\ell_{\text {last }}$ computed during $L_{i}^{a}$. In this case $L_{k}^{c}$ is a logical read action which also returns the value written by $L_{j}^{b}$. Consider the following cases:

Case 1: $L_{i}^{a}$ is enclosed free

By the induction assumption $L_{j}^{b}$ is the most recent write action serialized before $L_{k}^{c}$. If $L_{k}^{c}$ is serialized after $r_{i}^{a}[1] . s$ then since $L_{i}^{a}$ is serialized by $L_{k}^{c}$, we are done. Assume that $L_{k}^{c}$ is serialized before $r_{i}^{a}[1] . s$. According to Definition $10, L_{i}^{a}$ is serialized at $r_{i}^{a}[1] . s$. We claim that there are no good write actions serialized after $L_{k}^{c}$ and before $L_{i}^{a}$ (that is, before $\left.r_{i}^{a}[1] . s\right)$, and therefore the proof of this case follows. Assume by way of contradiction that there are such good writes. Let $\ell_{\ell}^{d}, \ell \leq w$, be the last label in $B_{H}^{r_{i}^{a}[1]} /(w+1)$. By this definition $L_{j}^{b} \Rightarrow L_{\ell}^{d}$. By Lemma $22 B_{H}^{r_{i}^{a}[1]} / i$ is a subgraph of $G_{i}^{a}$. Since $\ell_{\ell}^{d} \in B_{H}^{r_{i}^{a}[1]} /(w+1)$ we get that $\ell_{\ell}^{d} \in G_{i}^{a}$. In both of the following cases we reach the required contradiction:

Case 1.1: $\ell_{\ell}^{d} \in B_{i}^{a} / i$

Since $\ell_{k}^{c-r}$ is last in $B_{i}^{a} / i$, there is a path from $\ell_{k}^{c-r}$ to $\ell_{\ell}^{d}$ in $G_{i}^{a}$. Therefore by Theorem $23 L_{\ell}^{d} \Rightarrow L_{k}^{c-r}$. Since $r \geq 0$ we get that $L_{j}^{b} \Rightarrow L_{\ell}^{d} \Rightarrow L_{k}^{c}$, Hence, $L_{j}^{b}$ is not the last write action serialized before $L_{k}^{c}$, contradiction to the induction assumption. 
Case 1.2: $\ell_{\ell}^{d} \notin B_{i}^{a} / i$

In this case there is some label $\ell_{m}^{e}, m<\ell$, that excludes the suffix of $B_{H}^{r_{i}^{a}[1]} / i$ (and $\ell_{\ell}^{d}$ ) from $B_{i}^{a} / i$. Therefore $L_{\ell}^{d} \Rightarrow L_{m}^{e}$. Since $\ell_{m}^{e} \in B_{i}^{a} / i$ and $\ell_{k}^{c-r}$ is last in $B_{i}^{a} / i$, Theorem 23 implies that $L_{m}^{e} \Rightarrow L_{k}^{c-r}$. Therefore we get $L_{j}^{b} \Rightarrow L_{\ell}^{d} \Rightarrow L_{m}^{e} \Rightarrow L_{k}^{c-r} \Rightarrow L_{k}^{c}$ and in particular $L_{j}^{b} \Rightarrow L_{m}^{e} \Rightarrow L_{k}^{c}$, contradiction to the induction assumption.

Case 2: $L_{i}^{a}$ is not enclosed free

In this case $\ell_{k}^{c-r}$ is the label with the maximal $i d$ enclosed in $L_{i}^{a}$. By Definition 8 it holds that $r_{i}^{a}[1] . s \rightarrow r_{k}^{c-r}[1] . s$ therefore $L_{k}^{c-r}$ is serialized after $r_{i}^{a}[1] . s$. Since $r \geq 0$ we get that $L_{k}^{c}$ is serialized after $r_{u}^{a}[1] . s$. By the induction assumption, $L_{j}^{b}$ is the most recent write action serialized before $L_{k}^{c}$. Since $L_{i}^{a}$ is serialized by $L_{k}^{c}, L_{j}^{b}$ is the most recent write action serialized before $L_{i}^{a}$.

\section{ACKNOWLEDGMENTS}

We thank Paul Vitányi, for his tireless efforts to convince us that implementations with sublinear space complexity are worth looking at. We also thank Yael Gafni, Arie Rudich comments on an earlier version have helped us in this presentation. Last but surely not least is John Tromp whose continuous help during this work was invaluable in terms of both correctness and style.

\section{REFERENCES}

[Ab91a] U. Abraham, "MultiWriter Atomic Registers and bounded timestamps," preprint.

[Ab91b] U. Abraham, "On Interprocess Communication and the Implementation of a Multi-Writer Atomic Registers," preprint.

[CS90] R. Cori and E. Sopena "Some combinatorial aspects of Time Stamps Systems," submitted to J. Of Algorithms.

[IL87] A. Israeli, and M. Li, "Bounded Time-Stamps," Proceedings of the 28th Annual Symposium on Foundations of Computer Science, 1987, pp. 371-382.

[ILV87] A. Israeli, M. Li, and P. Vitányi, "Simple Multireader registers using Time-Stamp schemes," Report no. CS-R8758 Center for Mathematics and Computer Science, Amsterdam, Holland, November 1987.

[ITV92] A. Israeli, J. Tromp, and P. Vitányi, personal communication.

[La86a] L. Lamport, "On Interprocess Communication. Part I: Basic Formalism," Distributed Computing 1, 2 1986, pp. 77-85.

[La86b] L. Lamport, "On Interprocess Communication. Part II: Algorithms," Distributed Computing 1, 2 1986, pp. 86-101.

[LTV90] M. Li, J. Tromp, and P. Vitányi, "How to Share Concurrent Wait-Free Variables," Tech. Rept. CS-R8916, CWI, April 1989. Submitted to J.ACM/revision. (Prelim. Version in ICALP 89)

[LV90] M. Li and P. Vitányi, "Optimality of Wait-Free Atomic Multiwriter Variables," Submitted to Information Processing Letters 1990. 
[CS90] R. Cori and E. Sopena "Some combinatorial aspects of Time Stamps Systems," submitted to J. Of Algorithms.

[PB87] G.L. Peterson and J.E. Burns, "Concurrent reading while writing II: the multiwriter case", 28th Annual IEEE Symp. on Foundations of Computer Science, 1987, pp. 383-392.

[Sc89] R.W.Schaffer, "On the Correctness of Atomic Multi-Writer Registers", Technical Report MIT/LCS/TM-364, Laboratory for Computer Science, MIT.

[T92] J. Tromp, "On Update-Last Schemes", preprint.

[VA86] P. Vitányi, and B. Awerbuch, "Atomic Shared Register Access by Asynchronous Hardware," Proceedings of the 27th Annual Symposium on Foundations of Computer Science, 1986 , pp.233-243. 